\title{
ARTICLE OPEN \\ Charged grain boundary transitions in ionic ceramics for energy applications
}

\author{
K. S. N. Vikrant ${ }^{1}$ and R. Edwin García (D)
}

Surfaces and interfaces in ionic ceramics play a pivotal role in defining the transport limitations in many of the existing and emerging applications in energy-related systems such as fuel cells, rechargeable batteries, as well as advanced electronics such as those found in semiconducting, ferroelectric, and piezotronic applications. Here, a variational framework has been developed to understand the effects of the intrinsic and extrinsic ionic species and point defects on the structural and electrochemical stability of grain boundaries in polycrystalline ceramics. The theory predicts the conditions for the interfacial electrochemical and structural stability and phase transitions of charged interfaces and quantifies the properties induced by the broad region of electrochemical influence in front of a grain boundary capable of spanning anywhere from a few angtroms to entire grains. We demonstrate the validity of this theory for $\mathrm{Y}_{x} \mathrm{Zr}_{1-x} \mathrm{O}_{2-x / 2}$, cubic yttria stabilized zirconia. For small crystallographic misorientations, sharp Debye-type interfaces, $\mathrm{D}(1): \mathrm{C}^{V Y} \mathrm{~S}^{\underline{ }}$, are favored and promote high ionic conductivity in materials in polycrystalline form. For large grain boundary misorientations and large amounts of $\left[\mathrm{Y}_{2} \mathrm{O}_{3}\right]$ substitutions, three Mott-Schottky interfaces, MS(2): $\mathrm{C}^{V Y} \underline{\text { he }} \mathrm{S}^{V Y h e}, \mathrm{MS}(2)$ : $\mathrm{C}^{V Y h} \underline{\underline{h}} \mathrm{~S}^{e h V Y}$, and $\mathrm{MS}(2): \mathrm{C}^{V Y} \mathrm{~S}^{h e}$ are responsible for controlling grain boundary segregation and the observed poor macroscopic ionic transport, in great agreement with the scientific literature.

npj Computational Materials (2019)5:24; https://doi.org/10.1038/s41524-019-0159-2

\section{INTRODUCTION}

Over the last fifty years, a great deal of work describing the structural disorder, ${ }^{1-3}$ solute segregation, and associated mechanical properties, ${ }^{4,5}$ at the grain boundaries of polycrystalline materials has been made. ${ }^{6}$ Specifically, the thermodynamic formation of nanoscale intergranular thin films (IGFs), has been considered a class of interfacial adsorbates which exhibit structural properties that are spatially discernible and measurable at the boundary where two phases meet. ${ }^{1,7}$ Tang, Carter, and Cannon pioneered its description by starting from a phase field formulation and acknowledged that these two-dimensional interfacial features could undergo transitions, bifurcations, and stabilization/destabilization events as a result of externally applied thermal, structural, and chemical stimuli. ${ }^{8-10}$ These interfaces, defined as "complexions," do not adhere to the rigorous Gibbsian definition of phase, ${ }^{11}$ and its understanding has led to the development of an entire new field that has evolved into the rationalization and identification of discrete two-dimensional interfacial phases such as mono- and multilayered structures. ${ }^{12,13}$ Based on these ideas, the community has built design and thinking tools to rationalize technologically relevant mechanisms, including solid-state activated sintering and abnormal grain growth. ${ }^{10,14,15}$

The formation of interfacial phases in ionic ceramics has been reported to impact: (1) the chemistry and degree of ordering in the abutting crystals by changing the net charge accumulation at the interfacial core; and (2) the ion mobility along and across the interface by inducing undesirable interfacial structural or chemical disorder. ${ }^{16}$ In many of these materials, the formation of a charged region at a grain boundary has been recognized to be a result of the discontinuity of the properties of the ionic lattice, its associated Fermi level mismatch, and the difference in the adsorption (or segregation) of charged defects about the interfacial core. The resulting spatial distribution of ionic charge defines a set of thermodynamic Coulombic charge states that are inaccessible by its bulk counterpart, and further induces the electrostatic formation of a spatially varying electrostatic field, $\phi(\vec{x})$, as determined by Coulomb's law in its differential form, $\nabla \cdot \epsilon \nabla \phi=-\rho$. At elevated temperatures, the interfacial electrostatic (Schottky) potential, $\phi_{\circ}$, or the total net interfacial segregated charge, $\sigma_{\circ}$, contributes to define the thickness of the charged interface, as the grain boundary core thickness, $\delta$, determined by the chemical and structural interactions of the abutting phases plus the additional extent of the spatial charge, $\lambda$, to define the total extent of the interface, $L_{g b}=2 \lambda+\delta .{ }^{17}$ The resultant charged diffuse boundary defines a broad region of electrochemical influence, and can span from a few angstroms to an entire grain size, $S$, dramatically altering the macroscopic properties of the ionic ceramic. Analytical solutions found in the literature incorporate: (a) Guoy-Chapman-type descriptions to account for the effects of interfacial charge away from an infinitesimally thin grain boundary core; ${ }^{18-22}$ or (b) artificial corrections to the effect of the grain boundary thickness to fit the experimentally observed behavior of the charge layer through Mott-Schottky-type solutions. ${ }^{23-27}$ While existing descriptions correspond to piecewise solutions that are adapted by hand to meet specific applications in energy-related systems, ${ }^{25}$ the charged region has been demonstrated to control fundamental properties, such as electrical conductivity and ionic diffusivity. ${ }^{16,28,29}$ These properties can differ by several orders of magnitude with respect to the one displayed by a single-crystal

\footnotetext{
${ }^{1}$ School of Materials Engineering, Purdue University, West Lafayette, IN 47907, USA

Correspondence: R. Edwin García (redwing@alum.mit.edu)
}

Received: 18 August 2018 Accepted: 22 January 2019

Published online: 20 February 2019 

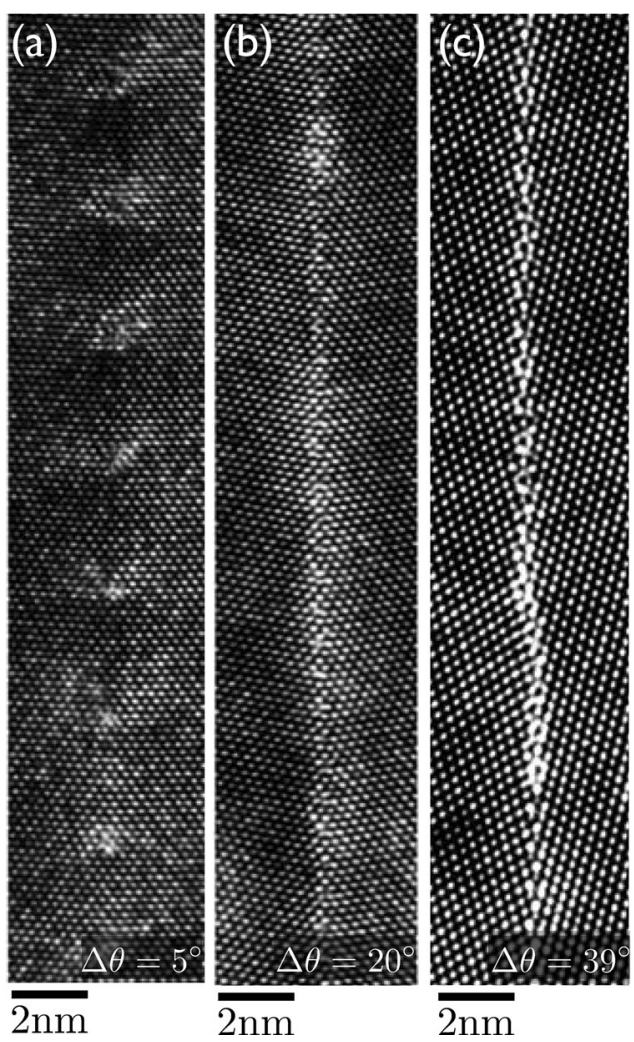

Fig. 1 High resolution TEM micrographs (insets a-c) for [110] symmetric tilt grain boundaries of $8 Y S Z$, as reported by Yoshida and co-workers. ${ }^{38}$ Results demonstrate that crystalline grain boundaries are favored for small misorientations; however, above a critical misorientation of $\Delta \theta_{c} \sim 20^{\circ}$, the grain boundary thickness abruptly changes from atomically sharp to $\delta \sim 1.3 \mathrm{~nm}$ leading to a disordered interfacial structure

sample at the same temperature. ${ }^{30}$ The optimization of these and other related properties in ceramic materials for electrochemical applications are key to the successful commercialization of emerging technologies, such as all-solid-state rechargeable batteries and low temperature solid oxide fuel cells, all of which are key elements for the development of a modern society based on renewable energy. Moreover, the identification and description of two-dimensional charged interfacial phases will allow an accurate rationalization of sintering of ceramic materials, ${ }^{31}$ positioning the scientific community to engineer mechanical or dielectric interfacial properties by tailoring the interfacial structural or chemical disorder.

One such material is zirconia based oxide ion conductor, which is one of the most prominently used materials for SOFCs electrolyte applications, oxygen sensors, and thermal barrier coatings. The ionic transport properties of $\mathrm{ZrO}_{2-x}$ is enhanced by stabilizing the cubic phase with the addition of $\mathrm{Y}_{2} \mathrm{O}_{3}$. The cubic phase is retained at $2000 \mathrm{~K}$ in the composition range of $5-25 \% .^{32}$ However, the total macroscopic electrical conductivity of polycrystalline Yttria Stabilized Ziconia, $\mathrm{Y}_{x} \mathrm{Zr}_{1-x} \mathrm{O}_{2-x / 2}, \mathrm{YSZ}$, is smaller than the corresponding single-crystal electrical conductivity due to the grain boundaries, which induce a blocking effect that is mainly associated to the excess oxygen vacancies at the grain boundary core and a space charge layer of depleted oxygen vacancies adjacent to the grain boundary. ${ }^{28}$ Electrical conductivity studies on YSZ carried by Dixon and co-workers, ${ }^{33}$ Strickler and Carlson, ${ }^{34}$ and Casselton, ${ }^{35}$ show that the total electrical conductivity of polycrystalline YSZ is increased with the increase of yttria in the system due to an increase in the average concentration of oxygen vacancies. The maximum conductivity for YSZ is obtained in the 7 to 9 mol\% of yttria substitution range, and in the $1000-1500^{\circ} \mathrm{C}$ temperature range. For larger amounts of yttria, oxygen vacancies encounter $Z r-Y$ and $Y-Y$ pairs that result in a decrease of mobility of oxygen vacancies, impacting the total ionic conductivity. ${ }^{36}$

For grain sizes larger than the total extent of the interface, $\mathcal{S}>L_{g b}$, grain boundary impedance studies on YSZ by Aoki and coworkers $^{37}$ demonstrate that segregation of yttrium and other extrinsic impurities such as silicon have a larger electrical resistance than those interfaces with none or low levels of dopants. HRTEM studies on YSZ bicrystals, ${ }^{38,39}$ show that the grain boundary interfacial energy and yttrium segregation is strictly dependent on the interfacial misorientation. Yoshida and coworkers show in Fig. 1a through c that the crystalline structure and local disorder of the grain boundary drastically changes as a function of crystallographic misorientation and suggests that yttrium segregation is more likely to be observed in large angle grain boundary misorientations. ${ }^{38,39}$ Atomistic simulations performed by Lee et al. support the coaccumulation of oxygen vacancies and yttrium at grain boundaries. ${ }^{40,41}$ The atomistic study also shows the formation of a thick oxygen vacancies depletion zone with a small yttrium depletion zone due to space charge effects in the vicinity of the interface. ${ }^{40,41}$

Great progress has been made in understanding the thermal and chemical effects on interfaces, including stoichiometric and electric-field effects in ferroelectric systems. ${ }^{42-46}$ However, the basic science of the effects that grain boundaries impose on their structural stability and resultant electrochemical phase transformations require further progress in order to improve on the design of the technological devices that they are part of. This paper provides a variational basis and a fundamental understanding on the impact of electrostatic charge and dipolar electrical energy on the thermodynamic stability of grain boundaries in ionic polycrystalline solids. This description enables the systematic research of charged interfaces and grain boundary transitions for ionic ceramics, including the chemical effects of dopant and point defects, such as ionic vacancies, interstitials, electrons, and holes, while naturally incorporating Maxwell's Equations. The method is demonstrated and validated for cubic Yttria Stabilized Zirconia, YSZ.

\section{RESULTS}

Theoretical framework

Define the Helmholtz free energy per unit volume, $f$, for a polycrystal as a function of the degree of crystallinity, $\eta$, and $N$ chemical species, $\left[V_{1}^{Z_{1}}\right], \ldots,\left[V_{N}^{Z_{N}}\right]$, including concentration of electrons, [e'], and holes, [h'], as classically defined. ${ }^{8}$ In the present study, the concentration of the electrons, $n$, is represented by $\left[\mathrm{e}^{\prime}\right]$ and the holes, $p$, by [h]. Here, $\eta$ is a coarse-grained measure of structural disorder, so that $\eta=1$ defines a perfectly crystalline lattice and $\eta=0$ defines a structurally disordered state. In addition, the $i$-th chemical component displays a valence, $Z_{i}$, which in turn contributes electrostatic energy from increasing the electrostatic charge, $\rho \phi$, and from increasing its dipolar moment density, $\frac{1}{2} \vec{D} \cdot \vec{E}$, to the system through the extended free energy, $f_{\circ}$, in agreement with Hart, ${ }^{47}$ Garca et al., ${ }^{48}$ and recent work: ${ }^{49}$

$$
\begin{aligned}
& f_{\circ}\left(\eta,\left[V_{1}^{Z_{1}}\right], \ldots,\left[V_{N}^{Z_{N}}\right],\left[e^{\prime}\right],\left[h^{\prime}\right], \rho, \vec{D}, T\right) \\
& \quad=f\left(\eta,\left[V_{1}^{Z_{1}}\right], \ldots,\left[V_{N}^{Z_{N}}\right],\left[e^{\prime}\right],\left[h^{\cdot}\right], T\right)+\rho \phi+\frac{1}{2} \vec{D} \cdot \vec{E}
\end{aligned}
$$

Equation (1) is a function of the coarse-grained charge density, $\rho$, the electric displacement vector, $\vec{D}$, and the position-dependent electric field, $\vec{E}$. In general, the resultant electric field is induced either by the surrounding electrostatic charge, or through the application of an external voltage difference. The spatial distribution of electrostatic charge raises the free energy of the system, $f_{\circ}$, 
when $\rho$ has the same sign as the voltage, $\phi$, at that same location. Thus, the second term on the right hand side of Eq. (1) favors the intermixing of spatial charge of opposite polarity, or promotes the local depletion of charge from those regions whose voltage has the same polarity as the charged species that attempts to locally occupy it.

The electrochemical free energy density, $f_{e c}$ of the system is defined through the Legendre transformation $f_{e c}\left(\eta,\left[V_{1}^{Z_{1}}\right], \ldots,\left[V_{N}^{Z_{N}}\right],\left[e^{\prime}\right],\left[h^{\cdot}\right], \vec{E}, T\right)=$

$f_{\circ}\left(\eta,\left[V_{1}^{Z_{1}}\right], \ldots,\left[V_{N}^{Z_{N}}\right],\left[e^{\prime}\right],[h], \vec{D}, T\right)-\vec{D} \cdot \vec{E}^{11,50}$ which specifies that when the material is electrostatically polarized, there is a decrease in free energy density, in agreement with several authors. ${ }^{48,49,51,52}$ In addition, for a constant magnetic field, the position-dependent electric field, $\vec{E}$, is a solution of Faraday's Law, $\nabla \times \vec{E}=\overrightarrow{0}$, thus, the voltage distribution, $\phi$, and the electric field are related through the relation, $\vec{E}=-\nabla \phi$. Finally, in the absence of ferroelectricity, the electric displacement vector and the electric field are related through the constitutive relation, $\vec{D}=\epsilon \vec{E}=-\epsilon \nabla \phi$.

Following the work of Kobayashi, Warren, and Carter, ${ }^{53}$ each single-crystal grain in a two-dimensional polycrystalline ionic solid is distinguished by an order parameter, $\theta$, which measures the local orientation of a crystal with respect to a fixed laboratory reference frame. The two abutting crystals develop a grain boundary which add an interfacial grain boundary energy penalty as a function of the local misorientation, $|\nabla \theta|$, and the local crystalinity, $\eta$, both of which are coupled through the monotonically increasing function $g(\eta)=\eta^{p}, p=\frac{3}{2}>1$. $s g(\eta)|\nabla \theta|$ corresponds to a monotonic increase in the grain boundary energy, which reduces to the classical Read-Shockley interface model in the limit of a sharp interface. ${ }^{53}$ In general, the grain boundary energy is a function of the three-dimensional orientation of the abutting grains. ${ }^{54-56}$ The formulation presented herein can be readily extended to include these effects, as reported by several authors for metallic systems, e.g., Pusztai and co-workers, ${ }^{54}$ Kobayashi and Warren, ${ }^{55}$ or Gránásy and coauthors. ${ }^{56}$ The sum of all the contributions to the total free energy functional is given by:

$$
\begin{aligned}
F & {\left[\eta, \theta,\left[V_{1}^{Z_{1}}\right], \ldots,\left[V_{N}^{Z_{N}}\right],\left[e^{\prime}\right],\left[h^{\prime}\right], \rho, \phi ; T\right] } \\
& =\int_{\Omega}\left[f\left(\eta,\left[V_{1}^{Z_{1}}\right], \ldots,\left[V_{N}^{Z_{N}}\right],\left[e^{\prime}\right],\left[h^{\prime}\right], T\right)+\rho \phi-\frac{\epsilon}{2}(\nabla \phi)^{2}\right. \\
& \left.+\frac{a^{2}}{2}(\nabla \eta)^{2}+s g(\eta)|\nabla \theta|\right] d \Omega
\end{aligned}
$$

Here, the first row accounts for the thermochemical and structural volumetric contributions to the system. The second row accounts for the electrical contributions as described in Eq. (1), for both the abutting grains and the interface. Finally, the third row corresponds to the contributions to grain boundary misorientation and their couplings to the local structural order/disorder of the interface. $s$ represents a structural coupling parameter as proposed by Warren and co-workers ${ }^{57}$ and $a$ is the gradient energy coefficient and quantifies the contributions to crystalline structural disorder gradients across a grain boundary. Equation (2) defines the equilibrium of a polycrystalline ceramic with electrostatically active grain boundaries by minimizing the free energy functional with respect to the controlling variables, $\left[V_{1}^{Z_{1}}\right], \ldots,\left[V_{N}^{Z_{N}}\right],\left[e^{\prime}\right],[h], \eta, \theta, \rho, \phi$. For ease in the description, volumetric, conserved or non-conserved, phase transformations such as chemical phase separation are not included; however, they can be easily incorporated, e.g., see. ${ }^{9,49,58-60}$ The local charge density imposes an additional physical constraint to the spatial distribution of the $i$-th species through the expression, $\rho=\sum_{i=1}^{N} e Z_{i}\left[V_{i}^{Z_{i}}\right] \cdot{ }^{48,49,51,52}$
The effect of solute and chemical defect interactions is incorporated by extending Tang's work for metallic, electrically neutral grain boundary complexions, ${ }^{9}$ and incorporating the effect of point defects by using standard defect equilibria concepts: ${ }^{50}$

$$
\begin{aligned}
f(\eta, & {\left.\left[V_{1}^{Z_{1}}\right], \ldots,\left[V_{N}^{Z_{N}}\right],\left[e^{\prime}\right],[h], T\right) } \\
= & \frac{1}{\nu}\left(\sum_{i=1}^{N}\left(f_{i}(\eta, T)\left[V_{i}^{Z_{i}}\right]+k_{b} T\left[V_{i}^{Z_{i}}\right] \ln \left[V_{i}^{Z_{i}}\right]\right)\right. \\
& +k_{b} T\left(1-\sum_{i=1}^{N}\left[V_{i}^{Z_{i}}\right]\right) \ln \left[1-\sum_{i=1}^{N}\left[V_{i}^{Z_{i}}\right]\right] \\
& \left.+\sum_{j=1, i \neq j}^{N} \Omega_{i j}\left[V_{i}^{Z_{i}}\right]\left[V_{j}^{Z_{j}}\right]\right) \\
& +\frac{a^{2}}{2} \eta^{2}(1-\eta)^{2}+\Delta H \frac{\Delta T}{T_{m}} p(\eta)
\end{aligned}
$$

where the free energy of formation contribution from the $i$-th component is $f_{i}(\eta, T)=f_{i}^{X}(T) p(\eta)+f_{i}^{S}(T)(1-p(\eta)) . f_{i}^{X}(T)$ is the free energy of formation in a perfectly crystalline lattice and $f_{i}^{S}(T)$ is the free energy of formation in the absence of crystalline ordering. $p(\eta)=\eta^{3}\left(6 \eta^{2}-15 \eta+10\right)$ is an interpolation function, in agreement with classic phase field formulations, ${ }^{53}$ and enables to distinguish spatial inhomogeneities of structural disorder, such as those occurring at the grain boundary. This spatial free energy variability favors the attraction of those ionic species and point defects whose energy of formation is lower than those displayed in structurally ordered regions. Local defect-defect interactions are incorporated into this formulation through the term on the third row of Eq. (3) and higher order interactions can be neglected or incorporated, depending on the analyzed material system at hand. For example, in YSZ, based on available first principles data $^{36}$ and molecular dynamic calculations, ${ }^{61}$ higher order interactions are reported to be negligible. However, higher order interactions can be easily incorporated by starting from fundamental thermodynamic principles, ${ }^{50,62,63}$ Equation (2) reduces to the work reported by Tang et al., ${ }^{9}$ in the limit of a binary, electrically neutral system.

The minimization of the thermodynamic variational principle described by Eq. (2) defines the general conditions of electrochemical-structural equilibrium through the variational derivatives:

$$
\begin{aligned}
\frac{\delta F}{\delta \eta} & =\frac{\partial f}{\partial \eta}-a^{2} \nabla^{2} \eta+s \frac{\partial g}{\partial \eta}|\nabla \theta|=0 \\
\frac{\delta F}{\delta \theta} & =-\nabla \cdot\left[s g(\eta) \frac{\nabla \theta}{|\nabla \theta|}\right]=0 \\
\frac{\delta F}{\delta \phi} & =\nabla \cdot \epsilon \nabla \phi+\rho=0 \\
\frac{\delta F}{\delta\left[V_{i}^{Z_{i}}\right]} & =\xi_{i}=\frac{\partial f}{\partial\left[V_{i}^{Z_{i}}\right]}+Z_{i} e \phi
\end{aligned}
$$

The first and second rows of Eq. (4) embody the structural equilibrium behavior of the grain boundary, as originally proposed by Kobayashi et al., ${ }^{53}$ and later by Tang et al. ${ }^{8}$ The third row of Eq. (4) corresponds to Coulomb's equation. ${ }^{48,51,52}$ Finally, the fourth row of Eq. (4) is identified as the electrochemical potential of the $i$ th ionic species, $\xi_{i}$ and reduces to its classic form in the absence of interfacial structural disorder, ${ }^{48,51,52}$ and to the chemical potential in the absence of structural and electrostatic charge, in agreement with textbooks. The electrochemical potential defines a generalized intensive local thermodynamic driving force for mass (and charge) segregation at interfaces as a result of gradients due to structural discontinuity, chemical potential, or electrostatic potential gradients.

In agreement with recent work, ${ }^{49}$ the equilibrium of the interface, $S$, in contact with a crystalline grain, $X$, is represented by equating their electrochemical potentials, i.e., $\xi_{i}^{S}\left(\eta=\eta_{\circ},\left[V_{i}^{Z_{i}}\right]=\left[V_{i}^{Z_{i}}\right]_{S}\right)=\xi_{i}^{X}\left(\eta=1,\left[V_{i}^{Z_{i}}\right]=\left[V_{i}^{Z_{i}}\right]\right)$, and results into $N$ equalities that relate the local concentration distribution of 


\section{4}

the $i$-th species, $\left[V_{i}^{Z_{i}}\right]$, to its interfacial electrical and structural state:

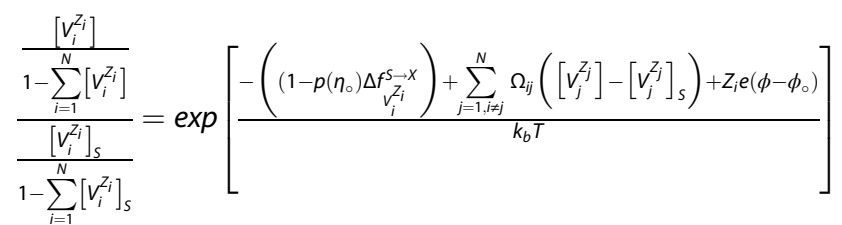

In contrast to existing analytical solutions, ${ }^{25-28}$ Eq. (5) naturally shows that segregation of the $i$-th component to a grain boundary is energetically favored by those charged species that have a higher free energy of formation in the crystalline phase as compared to the grain boundary, have a strong affinity to other already grain boundary segregating chemical species, when $\Omega_{i j}<$ 0 , or its electrostatic polarity is opposite in sign to the one displayed by the charged (grain boundary) interface. Thus, the total extent of the equilibrium charged grain boundary thickness naturally accounts for structural, chemical, and electrical contributions.

To understand the thermodynamic stability of a charged grain boundary, consider a single planar tilt misorientation centered at the origin of the laboratory reference system. The normal of the interface points along the $x$-axis, so its degree of crystallinity is $\eta(x=0)=\eta_{\circ}$, at the grain boundary core, and $\eta(x= \pm \infty)=1 \mathrm{far}$ away from the grain boundary, e.g., at the center of the crystalline grains. Following Tang and co-workers, ${ }^{8}$ the equilibrium orientation field is set to $\theta_{e q}(x)=\theta_{-}+\Delta \theta H(x)$, where $H(x)$ is the unit step function to quantify the crystallographic misorientation $\Delta \theta>0$ across the grain boundary core, so that $d \theta / d x=\Delta \theta \delta(x)$ contributes a localized contribution to Eq. (2) as a result of the grain boundary misorientation. $\delta(x)$ is the dirac function, as classically defined. Similarly, the equilibrium electrostatic charge per unit volume is defined as $\rho_{e q}(x)=\rho\left(\eta,\left[V_{1}^{Z_{1}}\right], \ldots,\left[V_{N}^{Z_{N}}\right],\left[\mathrm{e}^{\prime}\right],[\mathrm{h}]\right)+\sigma_{\circ} \delta(x)$, or alternatively by applying Gauss' theorem across the thickness of the grain boundary. ${ }^{64}$ Physically, the interfacial charge per unit area, $\sigma_{\circ}$, and the resultant spatial defect distribution in the abutting crystalline grains induces an electrostatic potential difference between the grain boundary and the far field crystal (grain) structure, i.e., the interfacial electrostatic potential, $\phi_{0}{ }^{25}$ Thus, the total free energy of the bicrystal is:

$$
\begin{gathered}
F\left[\eta,\left[V_{1}^{Z_{1}}\right], \ldots,\left[V_{N}^{Z_{N}}\right],\left[e^{\prime}\right],\left[h^{\prime}\right], \phi ; T\right]=s \Delta \theta g\left(\eta_{\circ}\right)+\sigma_{\circ} \phi_{\circ} \\
+2 \int_{0}^{\infty}\left[f\left(\eta,\left[V_{1}^{Z_{1}}\right],\left[V_{2}^{Z_{2}}\right], \ldots,\left[V_{N}^{Z_{N}}\right],\left[e^{\prime}\right],[h], T\right)\right. \\
\left.+\frac{a^{2}}{2}\left(\frac{d \eta}{d x}\right)^{2}+\rho \phi-\frac{\epsilon}{2}\left(\frac{d \phi}{d x}\right)^{2}\right] d x
\end{gathered}
$$

Substituting the charge neutrality condition, $\sigma_{\circ}+2 \int_{0}^{\infty} \rho d x=0$, and defining the total excess grain boundary energy as, $F_{x s}=F-$ $F_{\text {volumetric, }}$ it follows:

$$
\begin{aligned}
F_{x s}[\eta, & {\left.\left[V_{1}^{Z_{1}}\right], \ldots,\left[V_{N}^{Z_{N}}\right],\left[e^{\prime}\right],\left[h^{\cdot}\right], \phi ; T\right]=s \Delta \theta g\left(\eta_{\circ}\right) } \\
& +2 \int_{0}^{\infty}\left[\Delta f\left(\eta,\left[V_{1}^{Z_{1}}\right],\left[V_{2}^{Z_{2}}\right], \ldots,\left[V_{N}^{Z_{N}}\right],\left[e^{\prime}\right],\left[h^{\prime}\right], T\right)\right. \\
& \left.+\frac{a^{2}}{2}\left(\frac{d \eta}{d x}\right)^{2}+\rho\left(\phi-\phi_{\circ}\right)-\frac{\epsilon}{2}\left(\frac{d \phi}{d x}\right)^{2}\right] d x
\end{aligned}
$$

$F_{\text {volumetric }}$ is the total free energy of a single crystal in the limit of $\eta=1,\left[V_{i}^{Z_{i}}\right]=\left[V_{i}^{Z_{i}}\right]_{\infty}, \rho_{\infty}=0$. Thus, the excess chemical free energy density of an ionic solution, $\Delta f$, is given by:

$$
\begin{aligned}
\Delta f & \left(\eta,\left[V_{1}^{Z_{1}}\right], \ldots,\left[V_{N}^{Z_{N}}\right],\left[e^{\prime}\right],\left[h^{\cdot}\right], T\right) \\
& =f\left(\eta,\left[V_{1}^{Z_{1}}\right], \ldots,\left[V_{N}^{Z_{N}}\right],\left[e^{\prime}\right],[h], T\right) \\
& -f\left(\eta=1,\left[V_{1}^{Z_{1}}\right]_{\infty}, \ldots,\left[V_{N}^{Z_{N}}\right]_{\infty},\left[e^{\prime}\right]_{\infty},\left[h^{\cdot}\right]_{\infty}, T\right) \\
& -\sum_{i=1}^{N} \frac{\partial f\left(\eta=1,\left[V_{1}^{Z_{1}}\right]_{\infty}, \ldots,\left[V_{N}^{Z_{N}}\right]_{\infty},\left[e^{\prime}\right]_{\infty},\left[h^{\prime}\right]_{\infty}, T\right)}{\partial\left[V_{i}^{i_{i}}\right]}\left(\left[V_{i}^{Z_{i}}\right]-\left[V_{i}^{Z_{i}}\right]_{\infty}\right)
\end{aligned}
$$

The resultant electrochemical and structural fields correspond to interfacial state that minimize the interfacial free energy, $F_{x s}$, which explicitly quantifies the spatial deviations in free energy with respect to the equilibrium state of a single-crystal ionic solid, in agreement with Cahn, ${ }^{4}$ and Tang et al., ${ }^{9}$ as described by Eq. (7), and the corresponding variational derivatives:

$$
\begin{aligned}
\frac{\delta F_{x s}}{\delta \eta} & =-a^{2} \frac{d^{2} \eta}{d x^{2}}+\frac{\partial \Delta f}{\partial \eta}=0 \\
\frac{\delta F_{x s}}{\delta \phi} & =\epsilon \frac{d^{2} \phi}{d x^{2}}+\rho=0 \\
\frac{\delta F_{x s}}{\delta\left[V_{i}^{Z_{i}}\right]} & =\frac{\partial\left(\Delta f+\rho\left(\phi-\phi_{\circ}\right)\right)}{\partial\left[V_{i}^{Z_{i}}\right]}=0
\end{aligned}
$$

which includes the effect of misorientation, $\Delta \theta$ (first row of Eq. (4)), on the electrochemical and structural state of the interface. In agreement with Tang et al., ${ }^{9}$ and Cahn, ${ }^{4}$ multiply the first row of Eq. (9) by $\frac{\partial \eta}{\partial x^{\prime}}$ the second row by $\frac{\partial \phi}{\partial x^{\prime}}$ and the third row by $\frac{\partial\left[v_{i}^{Z_{i}}\right]}{\partial x}$. The resultant expressions are integrated and added in the limit of gradients vanishing away from the grain boundary, $\eta(x \rightarrow \infty)=1$, $\Delta f(\eta=1)=0$, and $\rho(\eta=1)=0$ :

$\frac{a^{2}}{2}\left(\frac{d \eta}{d x}\right)^{2}-\frac{\epsilon}{2}\left(\frac{d \phi}{d x}\right)^{2}=\Delta f+\rho\left(\phi-\phi_{\circ}\right)$

By using $g(\eta)=\int_{0}^{\eta} \frac{d g}{d \eta} d \eta$ and substituting Eq. (10) into Eq. (7), the excess free energy is written as a function of the integration variable, $\eta$, as:

$$
\begin{aligned}
& F_{X s}\left[\left[V_{1}^{Z_{1}}\right], \ldots,\left[V_{N}^{Z_{N}}\right],\left[e^{\prime}\right],[h], \phi ; \Delta \theta, \eta_{\circ}, T\right] \\
& \quad=2 \int_{0}^{\eta_{\circ}} \frac{s \Delta \theta}{2} \frac{\partial g}{\partial \eta} d \eta+2 \int_{\eta_{\circ}}^{1} \sqrt{2\left(\Delta f+\rho\left(\phi-\phi_{\circ}\right)\right)\left[a^{2}-\epsilon\left(\frac{d \phi}{d \eta}\right)^{2}\right]} d \eta
\end{aligned}
$$

in the absence of compositionally-induced phase transitions.

In agreement with Cahn for chemical systems, ${ }^{4}$ and later with Tang for structural-chemical systems, ${ }^{9}$ Eq. (11) demonstrates that structural disorder at the grain boundary core, $\eta_{0}$, is graphically identified as the sum of the two integrands of the functional (see Fig. 2a). Physically, the equilibrium interfacial crystalline order is a result of the direct competition between minimizing the interfacial free energy and the volumetric contributions to the excess chemical and electrostatic free energy in the abutting ionic ceramic grains. For a fixed excess chemical energy density, $\Delta f$, and fixed interfacial electrical energy, $\rho \phi$, small misorientations favor a crystallographically ordered interface, as represented by a single intersection of the volumetric and interfacial energy curves, as shown in Fig. 2a. As the misorientation increases, the degree of disorder monotonically increases (see Fig. 2b), until the grain boundary reaches a critical misorientation, $\Delta \theta \geq \Delta \theta_{c}$, at which point two types of equilibrium grain boundaries become energetically favorable (see Fig. 2b), one of which is crystallographically disordered and whose thickness is appreciably wider.

The interfacial electrical energy density contribution to the total free energy of the interface plays an important role on determining the structural stability of the grain boundary. For small misorientations, a positive increase of the interfacial electrical energy will favor an increase in the degree of structural order of the interface, while a negative contribution to the total free energy of the system will locally favor a small shift in the 
(a)

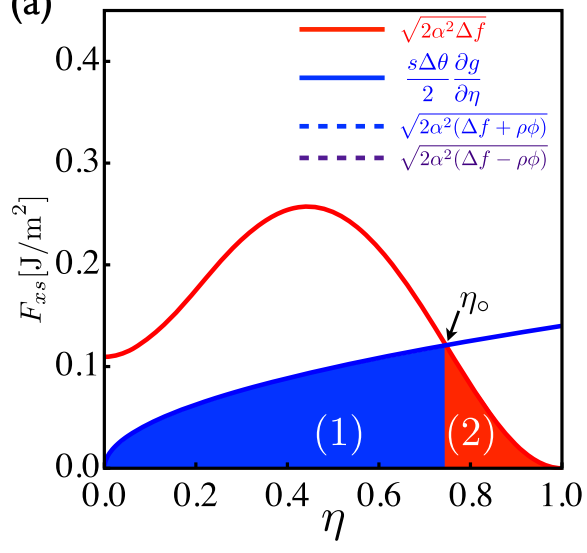

(b)
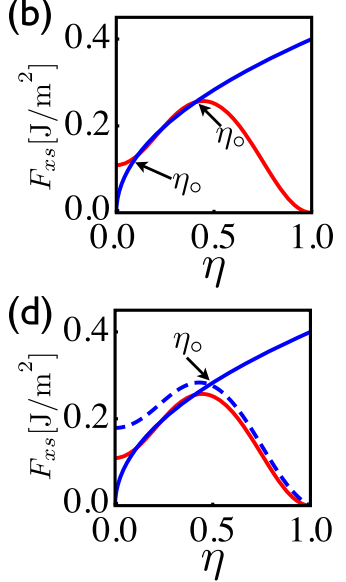
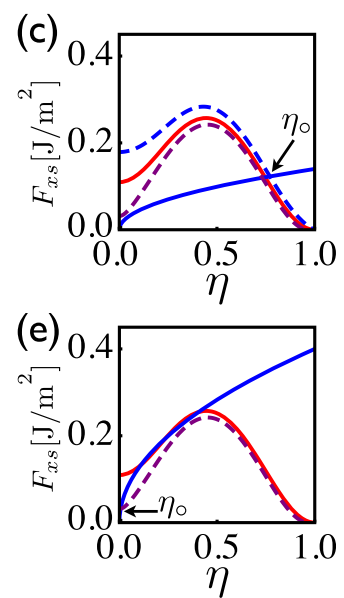

Fig. 2 Graphical description of interfacial degree of disorder as specified by Eq. (11). Inset a illustrates basic calculation, where the misorientation interfacial energy contribution is the area under the curve from zero to $\eta_{0}$ in blue (area (1)), and the volumetric free energy is the area from $\eta_{\text {。 }}$ to one, in red (area (2)). The total shaded area minimizes the interfacial excess free energy functional (see Tang ${ }^{8}$ or Cahn ${ }^{4}$ for a detailed description). Inset $\mathbf{b}$ shows that large misorientations can stabilize two interfacial states for fixed electrical energy density. Inset $\mathbf{c}$ shows that for small misorientations, the electrical energy of the interface can alter the structural disorder of the grain boundary (dashed blue corresponds to $\rho \phi>0$ and dashed purple to $\rho \phi<0$ ). For large misorientations, positive contributions to electrochemical energy can stabilize ordered grain boundaries (inset $\mathbf{d}$ ) and negative contributions to electrochemical energy will favor structurally disordered interfaces (inset $\mathbf{e}$ )

degree of interfacial disorder (see Fig. 2c). For intermediate and large misorientations, positive electrical contributions to the volumetric free energy of the system will shift the onset of the grain boundary transition, favoring more crystallographically ordered grain boundaries (see Fig. 2d). In contrast, negative contributions to the electrical energy of the grain boundary will favor crystallographically disordered interfaces, see Fig. $2 e$, thus enabling the early occurrence of an electrochemically-induced interfacial transition.

The interfacial electrochemical-structural equilibrium state is given by minimizing Eq. (11), and results in the Euler equations,

$$
\begin{array}{cc}
\frac{\delta F_{x S}}{\delta\left[V_{i}^{\mathcal{V}_{i}}\right]}=\frac{\partial\left(\Delta f+\rho\left(\phi-\phi_{0}\right)\right)}{\partial\left[V_{i}^{L_{i}}\right]}=\xi_{i}^{S}-\xi_{i}^{X} & =0 \\
\frac{\delta F_{x_{s}}}{\delta \phi}=2 \epsilon a^{2}\left(\Delta f+\rho\left(\phi-\phi_{\circ}\right)\right) \frac{d^{2} \phi}{d \eta^{2}}+\left[a^{2}-\epsilon\left(\frac{d \phi}{d \eta}\right)^{2}\right]\left(\epsilon \frac{\partial\left(\Delta f+\rho\left(\phi-\phi_{0}\right)\right)}{\partial \eta} \frac{d \phi}{d \eta}+a^{2} \rho\right) & =0
\end{array}
$$

subjected to the following boundary conditions at the grain boundary core: ${ }^{65}$

$$
\begin{aligned}
& s \frac{\Delta \theta}{2} \frac{\partial g}{\partial \eta}-\sqrt{2 a^{2}\left(\Delta f+\rho\left(\phi-\phi_{\circ}\right)\right)}=0 \\
& \quad \frac{d \phi}{d \eta}=0
\end{aligned}
$$

The first line of Eq. (12) represents the electrochemical potential as described by Eq. (4). The second line is Coulomb's Equation in its differential form as a function of the non-conserved order parameter, $\eta$, and describes the electrical state of the grain boundary. An alternate route to find Eq. (12) is to directly substitute Eq. (10) into Eq. (4), where $\left[V_{i}^{Z_{i}}\right]$ and $\phi$ are a function of $\eta$.

An asymptotic analysis on Eq. (12) demonstrates that for a disordered grain boundary core, $\eta \sim 0, \frac{\partial\left(\Delta f+\rho\left(\phi-\phi_{0}\right)\right)}{\partial \eta}=0$, and a nonzero interfacial potential, $\phi(\eta=0)$, the voltage $\phi \sim \phi(\eta=0)-\frac{a^{2} \rho_{o}}{2 \epsilon\left(\Delta f_{o}+\rho_{o} \phi_{c}\right)} \eta^{2}$, i.e., decays quadratically away from the charged interface. Here, $\phi_{c}$ corresponds to the interfacial potential at the critical misorientation, $\Delta \theta_{c}$. In addition, in the vicinity of the disordered grain boundary core, $\frac{\partial\left(\Delta f+\rho\left(\phi-\phi_{\circ}\right)\right)}{\partial \eta} \neq 0$, and thus, $\phi \sim \phi(\eta=0)+\phi_{+} \exp \left[\frac{\left(\Delta f_{\circ}+\rho_{o} \phi_{c}\right) \eta}{\frac{\partial\left(\Delta f+\rho\left(\phi-\phi_{o}\right)\right)}{\partial \eta}}\right]-\phi_{-} \exp \left[-\frac{\left(\Delta f_{\circ}+\rho_{\circ} \phi_{c}\right) \eta}{\frac{\partial\left(\Delta f+\rho\left(\phi-\phi_{o}\right)\right)}{\partial \eta}}\right]+$ $\frac{\rho a^{2} \eta}{\epsilon \frac{\partial\left(\Delta f+\rho\left(\phi-\phi_{0}\right)\right)}{\partial \eta}}$, which demonstrates that steep structural order-disorder gradients in the vicinity of the grain boundary can become a source or a sink of electric fields. Also,

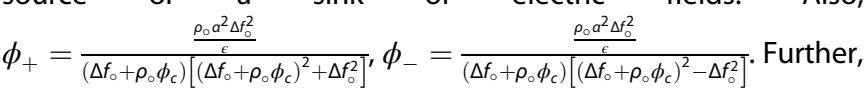
its spatial extent is equivalent to a Helmholtz-like layer and can shift the apparent interfacial potential.

Define $\Delta f=\Delta f_{\circ}(1-p(\eta))$ and $\rho=\rho_{\circ}(1-p(\eta))$ to describe the chemical energy density and the electrostatic charge density of the grain boundary to quantify accessible states that range between perfectly crystalline to completely disordered. Thus, Eq. (12) is written in dimensionless form:

$$
\begin{aligned}
(1 & -p(\eta))\left(1+\Pi_{1} \hat{\phi}\right) \frac{d^{2} \hat{\phi}}{d \eta^{2}} \\
& +\left(1-\Pi_{2}\left(\frac{d \hat{\phi}}{d \eta}\right)^{2}\right)\left(-\frac{p \prime(\eta)}{2}\left(1+\Pi_{1} \hat{\phi}\right) \frac{d \hat{\phi}}{d \eta}\right. \\
& \left.+\Pi_{1}(1-p(\eta))\left(\frac{d \hat{\phi}}{d \eta}\right)^{2}+(1-p(\eta)) \frac{\Pi_{1}}{2 \Pi_{2}}\right)=0
\end{aligned}
$$

two dimensionless numbers are identified:

$$
\begin{aligned}
\Pi_{1} & =\frac{\rho_{o} \phi_{c}}{\Delta f_{\circ}} \\
\Pi_{2} & =\frac{\epsilon \phi_{c}^{2}}{a^{2}}
\end{aligned}
$$

$\Pi_{1}$ embodies the contributions of electrostatic energy and chemical free energy as two driving forces that compete to induce electrostatic potential gradients in the vicinity of the interface. $\Pi_{2}$ corresponds to the competition between polarization electrical energy (voltage gradients), and the interfacial energy to form a disordered region (order parameter gradients). Thus, in the limit of $\Pi_{1} \rightarrow 0$ and $\Pi_{2} \rightarrow 0$, Eq. (14) reduces to the classic Coulomb's Equation.

Figure 3 summarizes the solutions to Eq. (14) for selected physical dimensionless number values, $\Pi_{1}$ and $\Pi_{2}$, and describes the possible interfacial electrochemical properties as a function of the structural state of the interface. Specifically, Fig. 3a shows that if the local interfacial chemical and electrical energy have the same polarity, then the interfacial potential will be positive. In addition, for material systems where the electrical energy is comparable in order of magnitude to the free energy contributions to defect formation and mixing at an interface, the interfacial potential can undergo changes in polarity as the order parameter 
(a)

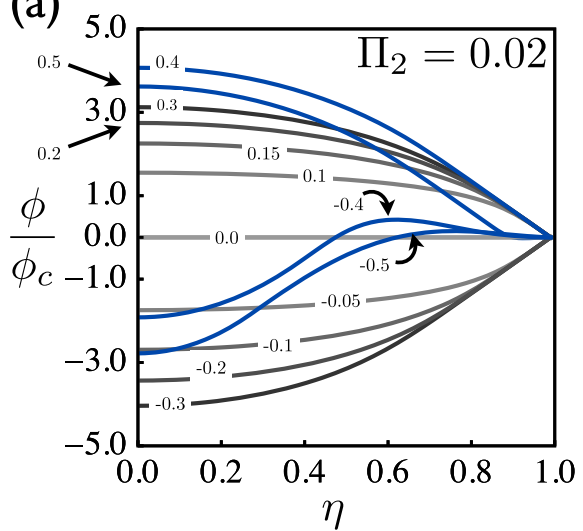

(b)

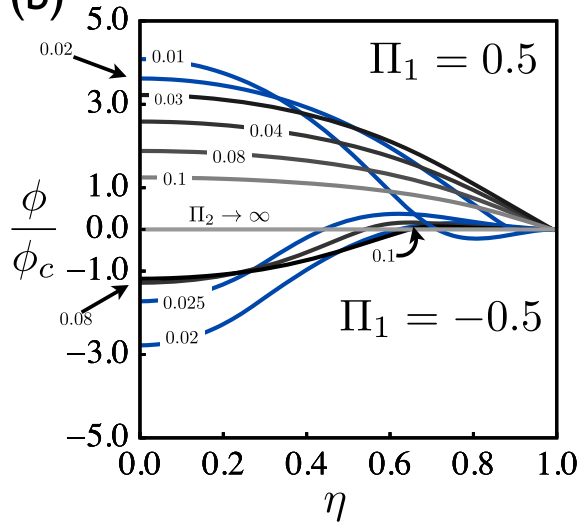

Fig. 3 Interfacial electrostatic potential, $\phi$, as a function of the structural state, $\eta$, of the interface for selected dimensionless numbers. Inset a shows the effects of the electrical and chemical energy on the interfacial potential and highlights conditions to tailor the electrical polarity of an interface or the conditions to make it electrically neutral. Here, each label highlights the $\Pi_{1}$ value associated to each curve. Inset $\mathbf{b}$ demonstrates the effects of the polarizability of the interface and the interfacial energy penalty of a disordered region. Here, each label highlights the $\Pi_{2}$ value associated to each curve. See text for details

changes from perfectly disordered to crystalline, thus suggesting that for $\left\|\Pi_{1}\right\|>0.3$, the range of misorientations delivering negligible interfacial potential values is wider, particularly for $\Pi_{1}$ $<-0.3$. Also, because the charge transport properties of polycrystalline ceramics are known to be controlled by the amount of segregated surfactant and defects available at the interface, Eq. 15 suggests that doping a polycrystalline ceramic can readily be used to tailor the electrical transport properties for both homo- and heterointerfaces. Thus, the ionic transport of polycrystalline ceramics that possess a strongly insulating behavior can be improved by introducing an ionic species or point defect that favors the formation of a crystalline interface, or alters the sign of the interfacial electrostatic potential to lightly attract the controlling point defect and enables the transport of the primary charge carrier but does not lock it in to the interface.

Figure $3 \mathrm{~b}$ shows that for materials where the electrical driving forces are comparable to the chemical driving forces for segregation, e.g., $\Pi_{1}= \pm 0.5$, the magnitude of the interfacial potential is maximized for materials that have a higher dielectric susceptibility and display a small interfacial energy penalty to form a structurally disordered interface. Further, the range of misorientations that display zero interfacial potential increases for materials having higher dielectric susceptibility.

Away from this range of dimensionless numbers, the interfacial potential decreases asymptotically, particularly if the interfacial energy necessary to form a structurally disordered interface is comparable or higher than the energy to induce an interfacial voltage gradient. For small angle misorientations, interfaces will develop a non-zero potential. This is not desirable, particularly for those applications where small angle grain boundary misorientations are favored during processing.

\section{Application to cubic YSZ}

For fixed misorientation, interfacial composition, and electrostatic interfacial potential, the graphical construction associated to Eq. (11) allows to specify the equilibrium interfacial crystallographic disorder, while the solutions to Eq. (12) allow to specify the interfacial potential, $\phi$, as a function of the order parameter, $\eta$, for fixed misorientation. Both descriptions independently provide a family of solutions to the electrochemical and structural state of the interface. Specifically, Fig. 4a shows the graphical solutions of Eq. (11) in agreement with Tang and co-workers, ${ }^{9}$ for selected misorientation values, for 8 YSZ at $2000 \mathrm{~K}$ (see section Numerical Implementation for details). Figure $4 \mathrm{~b}$ shows that as the misorientation increases, the range of $\eta$ values that are accessible by the interface decreases, thus shifting toward more disordered states.

The solutions of Eq. (11) indeed minimize the energy of the interface, but fail to satisfy Maxwell's equations. The overall equilibrium state of the interface is a result of minimizing Eq. (11) while simultaneously satisfying Eq. (12). Figure 5 (red line) shows the interfacial equilibrium potential as predicted by Eq. (14) alone as a function of the interfacial degree of crystallinity, $\eta$, for 8YSZ. Here, $\Pi_{1}=0.5$ and $\Pi_{2}=0.02$, as shown in Fig. 3a. The equilibrium interfacial electrical and structural state is shown in Fig. 5 as the location(s) where both solutions intersect for selected misorientation values, $\Delta \theta=15,20.3$, and $30^{\circ}$. Results demonstrate that for small misorientations, e.g., $\Delta \theta=15^{\circ}$, the interfacial electrostatic potential is single-valued and asymptotically increases as the grain boundary misorientation increases, until a critical misorientation, $\Delta \theta=\Delta \theta_{c}=20.3^{\circ}$ is reached, where two solutions (intersections) are identified, and thus both interfacial states can coexist. For $\Delta \theta>\Delta \theta_{c}$, the solution becomes single valued again, and defines an interfacial structural and electrostatic first order transition, i.e., a charged grain boundary transition, from a low to high degree of interfacial structural disorder, and from a low to high interfacial electrostatic potential.

Above the critical misorientation, $\Delta \theta_{c}=20.3^{\circ}$, for example, $\Delta \theta=30^{\circ}$, the grain boundary exhibits a structurally disordered grain boundary state, and the Schottky potential reaches an asymptotic maximal value. The structural thickness of the grain boundary is estimated as $\delta \sim \int_{-L}^{L}(1-\eta) d x$, in agreement with Warren and co-workers. ${ }^{57}$ Further, for small angle grain boundary misorientations, the grain boundary thickness is $\delta \sim 0.8 \mathrm{~nm}$ and undergoes an interfacial transition to $\delta \sim 1.9 \mathrm{~nm}$ above the critical misorientation value. This result is in perfect agreement with TEM experiments from Yoshida and co-workers (see Fig. $1 \mathrm{a}-\mathrm{c}$ ), ${ }^{38}$ and demonstrates that the state of the interface is a result of simultaneously minimizing the interfacial energy of the interface and satisfying Maxwell's Equations.

The predicted spatial distribution of point defects in the vicinity of a grain boundary for $8 \mathrm{YSZ}$, at a misorientation $\Delta \theta=15^{\circ}$ is shown for Fig. 6a. Here, when the structural grain boundary is atomically sharp and it is sandwiched by a single, diffuse charge layer, it is defined herein as of Debye-type. For this grain boundary, $\delta \sim 0.8 \mathrm{~nm}$, it is positively charged (see Fig. $6 \mathrm{~d}$ ), and its core $(C)$ is rich in oxygen vacancies and yttrium defects, in perfect agreement with experimental results. ${ }^{28}$ The positively charged grain boundary induces the formation of an oxygen vacancy-rich Debye-type (D) depletion zone $0.5 \mathrm{~nm}$ away from the 

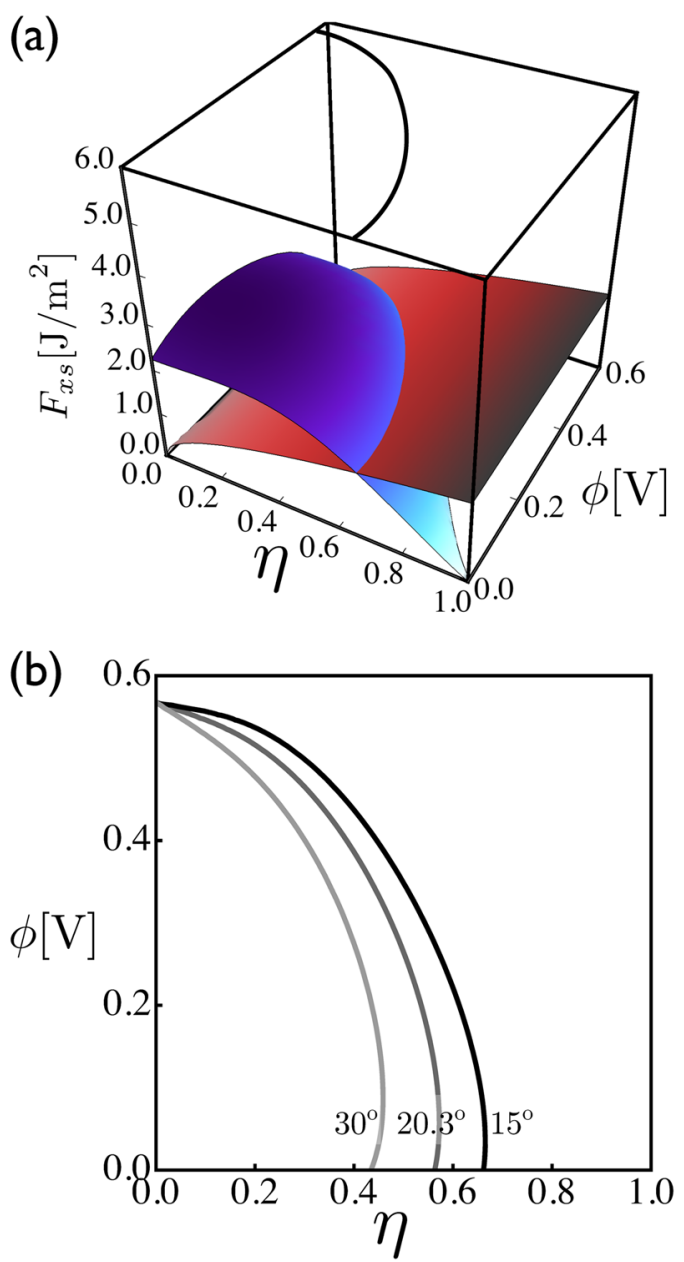

Fig. 4 Graphical construction of the interfacial free energy as described by Eq. (11) to determine charged grain boundary states $\eta, \phi$ for selected grain boundary misorientations, $\Delta \theta$, as shown in inset a for 8YSZ. The intersection of volumetric free energy density surface (in shades of blue) with misorientation interfacial energy density surface (in shades of red) defines a subset of electrostatic potential and crystallographic disorder values that minimizes the energy of the interface, but ignores the electrostatic energy contributions. Inset b shows $\phi(\eta)$, for selected misorientations. The shade of gray changes from dark to light with increase in misorientation values for $\Delta \theta=15^{\circ}$ (darkest), $\Delta \theta=20.3^{\circ}, \Delta \theta=30^{\circ}$ (lightest)

interface and has an extent of $\sim 1.5 \mathrm{~nm}$. The accumulation of electrons and depletion of holes is negligible because it deviates from the single-crystal concentration less than $1 \%$.

For intermediate misorientations, e.g., $\Delta \theta=20^{\circ}$ (see Fig. 6b), the grain boundary structural thickness and the degree of disorder monotonically increases, making the interface more favorable for oxygen vacancies and electrons. This increase in the positive electrostatic charge at the grain boundary extends the depletion zone of oxygen vacancies in front of the grain boundary as shown in Fig. 6e. In addition, a small depletion zone of yttrium defects occurs as a result of the attraction of yttrium defects and oxygen vacancies, in agreement with atomistic calculations ${ }^{40,41}$ and experimental results by Aoki and co-workers. ${ }^{37}$ The resultant thicker structurally disordered, grain boundary core space charge that is sandwiched by a wide diffuse charge layer defines a Mott-Schottky-type interface, ${ }^{26,27}$ (MS), and it is a natural consequence of the electrochemical and structural interactions between the grain boundary and the abutting grains.

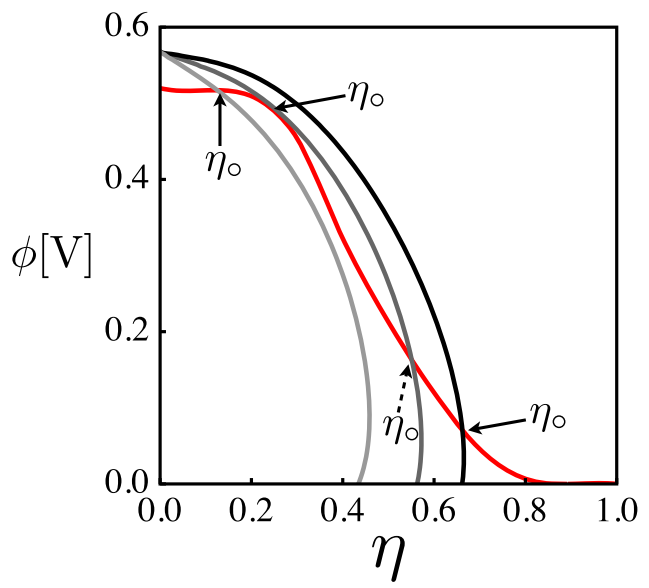

Fig. 5 Graphical construction depicting charged grain boundary transition as a function of interfacial degree of crystallinity. Equation (14) solutions is shown as a red line. Equation (11) solutions are shown for fixed $\Delta \theta=15^{\circ}$ (dark gray), $\Delta \theta_{c}=20.3^{\circ}$ and $\Delta \theta=30^{\circ}$ (light gray), and graphically demonstrates that the structural crystallinity and interfacial electrostatic potential of the interface that simultaneously minimizes Eq. (11) and satisfies Maxwell's Equations corresponds to the intersection of the red curve with a gray line. Thus, for: a small misorientations (black) small interfacial potentials and crystalline grain boundaries are favored; $\mathbf{b}$ at the charged grain boundary transition misorientation, a two electrochemical-structural interfacial states are stable (dark gray); and for high misorientations, large interfacial potentials and low degree of crystallinity is energetically favored

For $\Delta \theta>\Delta \theta_{c}=20.3^{\circ}$, e.g., $\Delta \theta=30^{\circ}$, the grain boundary thickness abruptly increases to $\delta \sim 2.1 \mathrm{~nm}$, further favoring more oxygen vacancies in the grain boundary core, and extends the oxygen vacancies depletion zone thickness in front of the grain boundary to $\sim 2.8 \mathrm{~nm}$ (see Fig. $6 \mathrm{c}-\mathrm{f}$ ). The yttrium segregation at the grain boundary has greatly increased by depleting the available solute from the immediate surroundings to a value well beyond the far field composition. Furthermore, the $\left[\mathrm{e}^{\prime}\right]$ accumulation thickness extends beyond the $\left[V_{0}^{\prime \prime}\right]$ depletion zone and has a thickness of $\sim 4.5 \mathrm{~nm}$, while an [h'] depletion zone develops with a thickness of $\sim 4 \mathrm{~nm}$, making this region effectively $\mathrm{n}$-doped. Overall, the resultant charged interfacial state is MS-type and because of the low ionic mobility of yttrium and oxygen vacancies at the interface, it will result in an ionically insulating grain boundary. Results demonstrate that yttrium segregates at grain boundaries in small amounts, and the segregated amount is highly dependent on the misorientation. ${ }^{38}$

The effect of yttria doping on the electrochemical grain boundary state is shown in Fig. 7. For a fixed grain boundary misorientation, $\Delta \theta=20^{\circ}$, results demonstrate that as the macroscopic concentration of yttria increases, the disorder of the interface increases favoring thicker grain boundaries (see Fig. 7a). Further, the grain boundary core $\left[V_{o}^{\cdot}\right]$ increases by a factor of 1.5 as the average amount of yttrium increases (see Fig. 7b), even though the oxygen vacancies depletion region in the vicinity of the grain boundary significantly decreases from a one order of magnitude difference to an experimentally negligible amount because as the bulk amount of yttria increases, the grain boundary does not need to deplete $\left[Y_{\mathrm{Zr}}^{\prime}\right]$ from the vicinity of the interface. The spatial extent of $\left[Y_{\mathrm{Zr}}^{\prime}\right]$ decreases from a $\sim 4$ to $\sim 1 \mathrm{~nm}$ wide region near the grain boundary and corresponds to an MS-type interface. In addition, the effective increase in positive electrostatic charge at the grain boundary core suppresses the hole concentration and enhances the electron concentration (see Fig. 7c) nearly by one order of magnitude, thus making the grain boundary core slightly $n$-doped. The [h'] depletion zone thickness 

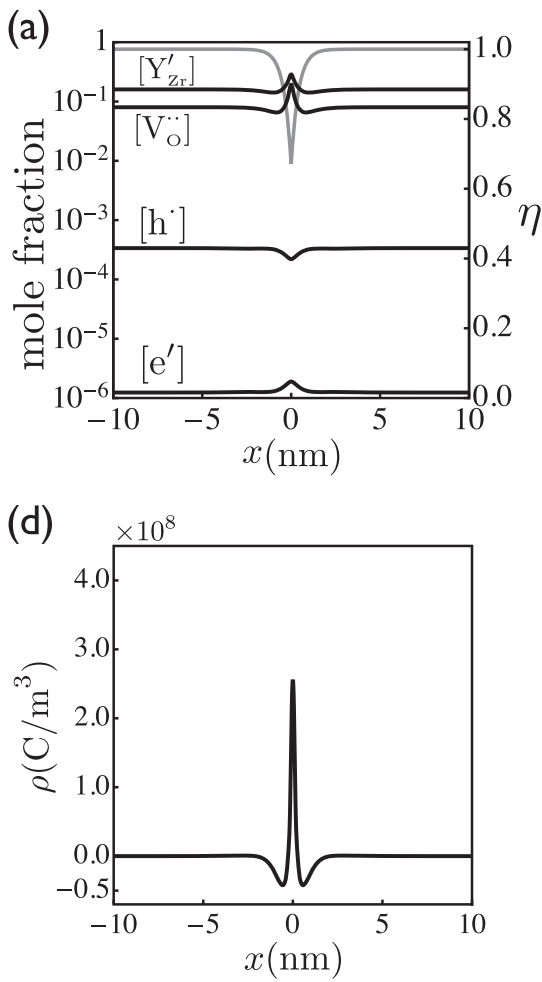
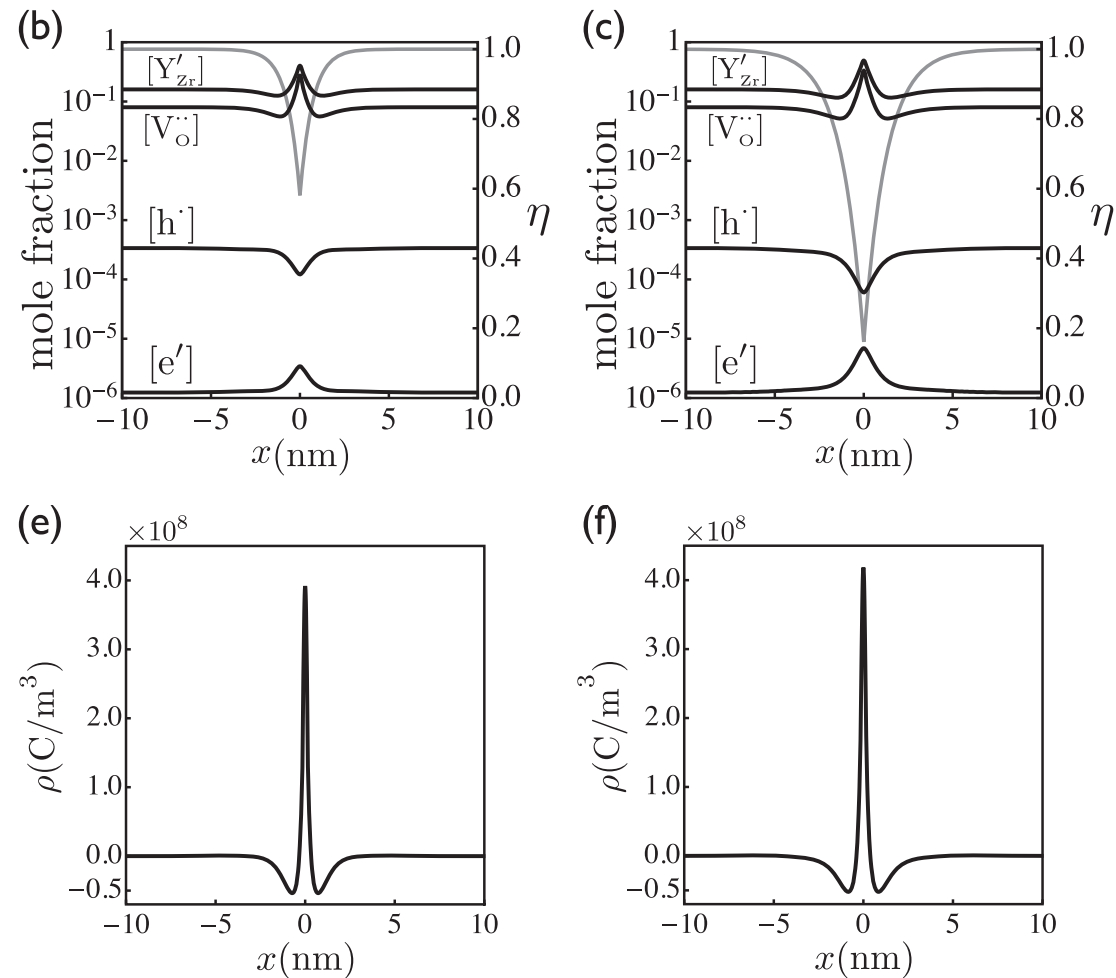

Fig. 6 Predicted defect distribution and degree of crystallinity in the vicinity of the grain boundary for selected misorientations, $\Delta \theta=15^{\circ}$ (inset a), $\Delta \theta=20^{\circ}$ (inset $\mathbf{b}$ ), and $\Delta \theta=30^{\circ}$ (inset $\mathbf{c}$ ). The grain boundary is located at $x=0$. The corresponding charge density corresponds to $\Delta \theta=15^{\circ}$ (inset $\mathbf{d}$ ), $\Delta \theta=20^{\circ}$ (inset $\mathbf{e}$ ), and $\Delta \theta=30^{\circ}$ (inset f). The grain boundary core is positively charged as a result of [ $\left.V_{\mathrm{o}}^{\prime \prime}\right]$ being chemically attracted to the interface, depleting the point defects in the vicinity of the surrounding grains. In turn, a positive electrostatic interfacial potential develops at the grain boundary. The resultant electrochemical state induces the interfacial attraction of $\left[Y_{\mathrm{Zr}}^{\prime}\right]$ and [e'] due to its opposite charge polarity and the repulsion of [h'] in agreement with experimental results ${ }^{17,23,28}$

increases from 1 to $6.5 \mathrm{~nm}$, while [e'] accumulation zone thickness increases from 1 to $7 \mathrm{~nm}$ in the grain boundary core. Overall, calculations demonstrate that for large amounts of yttria, $\left[V_{0}\right]$ and $\left[Y_{\mathrm{Zr}}^{\prime}\right]$ in the grain boundary core increases, while $\left[V_{\mathrm{o}}^{\prime \prime}\right]$ and $Y_{\mathrm{Zr}}^{\prime}$ depletion region shrinks in the immediate surroundings of the grain boundary.

\section{DISCUSSION}

We have developed a continuum thermodynamic framework that simultaneously describes the structural and electrochemical character of a grain boundary. Here, the structural state of the interface is directly coupled to the electrochemical state, so that charged grain boundaries that display an opposite polarity with respect to the dominant charge species (or point defects) are more likely to be disordered. Specifically, the electrical contribution to the excess free energy of an interface leads to the natural development of charged interfacial state whose interfacial degree of disorder increases with misorientation so that: (a) low misorientations and small solute concentrations of point charge defects favor Debye-type (D) interfaces; (b) intermediate and high misorientations favor Mott-Schottky-type (MS) interfaces; (c) below the charge grain boundary transition, $\Delta \theta<\Delta \theta_{c}$, mono-(D) and bilayer (MS) interfacial structures are stabilized; (d) above the interfacial transition, $\Delta \theta>\Delta \theta_{c}$ thicker bilayer MS interface transitions are spontaneously stabilized; finally, (e) large amounts of doping can completely suppress the abrupt appearance of the charge interface transition, and instead favor the uniform increase of the extended thickness of the interface, leading to interfacial electrochemical states that greatly differ from the average values.
The effects of yttria doping and grain boundary misorientation are summarized in a grain boundary phase diagram, see Fig. 8. Here, charged grain boundaries are classified as $T(N)$ : $C^{V_{1} V_{2} \ldots V_{M}} S^{V_{1} V_{2} \ldots V_{P}}$, where $T$ defines the electrochemical character of the grain boundary thickness as of Debye-type (D), or Mott-Schottky-type (MS). The state of the grain boundary core, $C$, is specified by the super-index $C^{V_{1} V_{2} \ldots V_{M}}$ and defines the excess or depleted chemical components at the core, while $S^{V_{1} V_{2} \ldots V_{P}}$ defines the composition of the layers in the vicinity of the interface. $\mathrm{N}$ defines the total number of spatially distinguishable layers based on composition in the vicinity of the interface, in agreement with the scientific literature. ${ }^{22,26,27,66,67}$ Underlined compositions, $V_{i}$, correspond to a depleted component. Specifically, for YSZ, define $V=\left[V_{\mathrm{O}}^{*}\right], Y=\left[Y_{\mathrm{Zr}}^{\prime}\right], e=\left[\mathrm{e}^{\prime}\right]$, and $\mathrm{h}=\left[\mathrm{h}^{\prime}\right]$ shorthand notation. The layer thickness of each charged species, $\left[V_{\mathrm{O}}^{\prime \prime}\right],\left[Y_{\mathrm{Zr}}^{\prime}\right],\left[\mathrm{e}^{\prime}\right]$, and $\left[\mathrm{h}^{\prime}\right]$ is defined herein as the spatial extent away from the interface where the system deviates from the average (far field) concentration by $1 \%$.

Small angle misorientations favor structurally ordered grain boundary states. The core mainly consists of $\left[Y_{\mathrm{Zr}}^{\prime}\right]$ and excess $\left[V_{\mathrm{O}}^{\prime \prime}\right]$ resulting in positively charged grain boundaries. Intermediate misorientations, below the charged grain boundary transition, for yttria dopants less than $14.5 \%$, D-states continuously transform to bilayer (MS(2): $\mathrm{C}^{\text {VYhe }} \mathrm{S}^{\mathrm{VYhe}}$ ) interfacial states because of an increase in disorder at the grain boundary core.

The yttria dependent critical misorientation, $\Delta \theta_{c}\left(\left[\mathrm{Y}_{2} \mathrm{O}_{3}\right]\right)$ above which the first order charged interfacial transition is observed, decreases as the amount of yttria content increases, and completely disappears at critical composition, $\left[\mathrm{Y}_{2} \mathrm{O}_{3}\right]_{c} \sim 14.5 \%$ (solid line in Fig. 8). For $\left[\mathrm{Y}_{2} \mathrm{O}_{3}\right]<\left[\mathrm{Y}_{2} \mathrm{O}_{3}\right]_{C}$, the grain boundary 

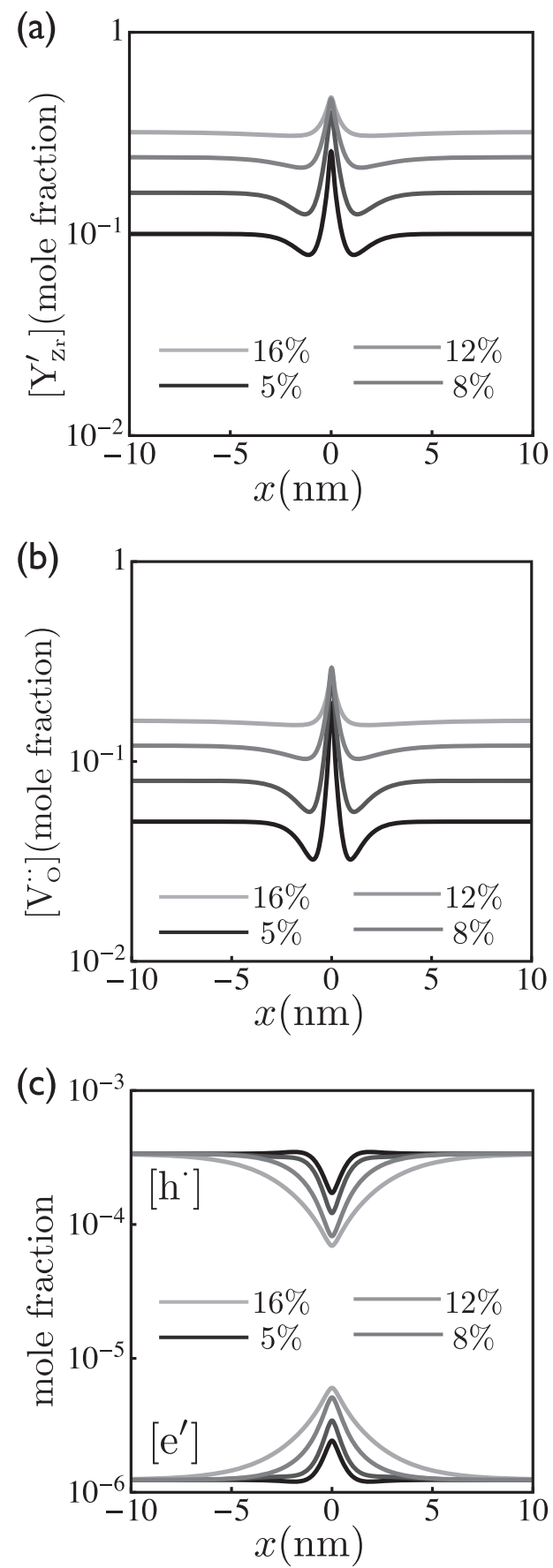

Fig. 7 Effect of yttria doping on the spatial distribution of point defects for a grain boundary misorientation $\Delta \theta=20^{\circ}$. Inset a shows the $\left[V_{\mathrm{o}}^{\prime \prime}\right]$ distribution, $\mathbf{b}$ shows $\left[Y_{\mathrm{Zr}}^{\prime}\right]$ distribution, and $\mathbf{c}$ shows $\left[\mathrm{h}{ }^{\prime}\right]$ and $\left[e^{\prime}\right]$ distribution in the vicinity of the grain boundary. The shade of gray changes from dark to light with yttria dopant concentration, $5 \mathrm{~mol} \%$ (darkest), 8, 12, and $16 \mathrm{~mol} \%$ (lightest)

thickness, structural order, and the associated interfacial electrostatic potential undergoes a first order phase transition resulting in thick, electrically insulating grain boundary cores with bilayer charged interfaces, $\left(\mathrm{MS}(2): \mathrm{C}^{\mathrm{VYhe}} \mathrm{S}^{\text {ehVY }}\right)$. For $\left[\mathrm{Y}_{2} \mathrm{O}_{3}\right]>\left[\mathrm{Y}_{2} \mathrm{O}_{3}\right]_{c}$, the charged layers continuously undergo the transition from $D(1)$ : $\mathrm{C}^{V Y} \mathrm{~S}^{\underline{Y}} \rightarrow \mathrm{MS}(2): \mathrm{C}^{V Y} \mathrm{~S}^{\underline{h} e}$, and suggest a wide range of misorientations and large yttria doping that the material has to go through to develop thick, electrically insulating grain boundaries.

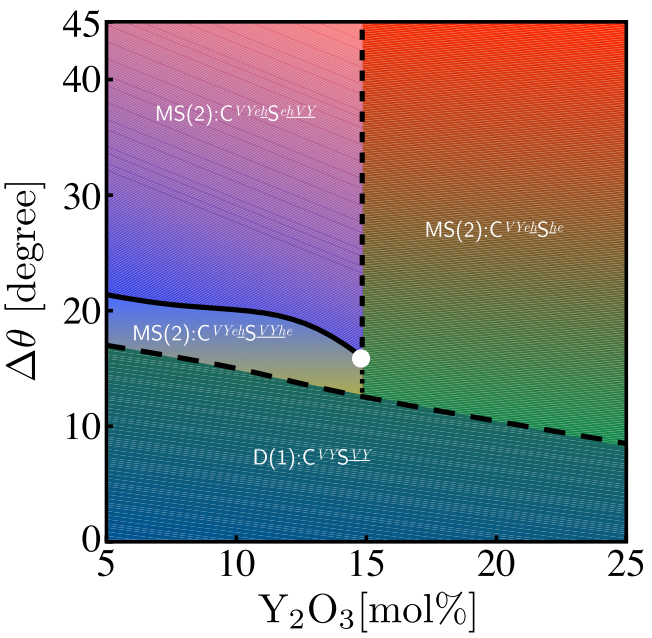

Fig. 8 Grain boundary phase diagram at $2000 \mathrm{~K}$. For YSZ, the grain boundary cores are positively charged due to an excess of $\left[V_{0}^{\prime \prime}\right]$. Small angle grain boundary misorientations favor the formation of Debye-type (D) interfaces that display a single charged layer. Intermediate angle grain boundary misorientations increases the interfacial disorder, the thickness of grain boundary and the space charge layers favoring Mott-Schottky-type (MS) interfaces. Ordered and disordered grain boundaries coexist in the 5 to $14.5 \% \mathrm{Y}_{2} \mathrm{O}_{3}$ composition range for $\Delta \theta_{c}=\Delta \theta_{c}\left(\left[\mathrm{Y}_{2} \mathrm{O}_{3}\right]\right)$, exhibiting a first order structural transition (thick black line). Above $14.5 \%$ there is no first order structural transition. Large angle grain boundaries develop a thick $\left[\mathrm{e}^{\prime}\right]$ accumulation zone and $\mathrm{a}[\mathrm{h}$ ] depletion zone resulting in wide (MS) bilayer interfaces

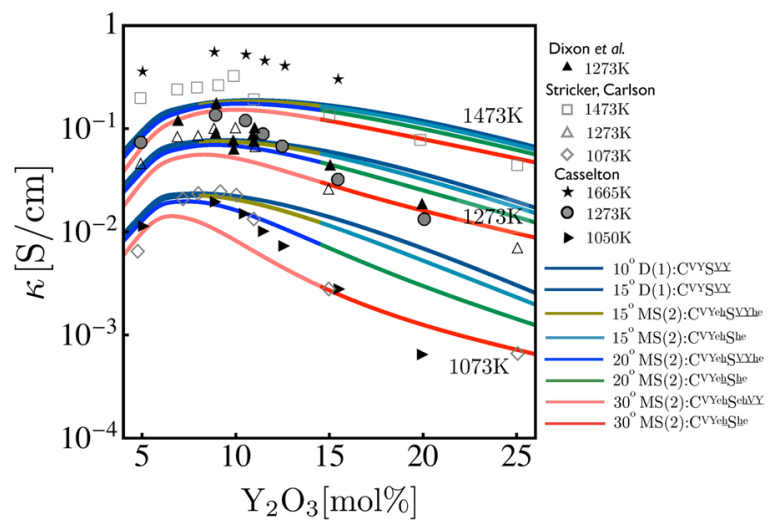

Fig. 9 Comparison of experimentally measured versus simulated total electrical conductivity for YSZ, for $\sim 1 \mu \mathrm{m}$ grain size polycrystalline data. The effect of yttria content on experimental electrical conductivity corresponds to data measured at 1073, 1273, and $1473 \mathrm{~K}$, as reported by Dixon and co-workers, Strickler, and Carlson, and Casselton. ${ }^{33-35}$ Large angle, MS-type grain boundaries exhibit lower total conductivity than small angle, D-type and intermediate angle MS-type grain boundaries due to a thick grain boundary core, and wide depletion zone of $\left[V_{0}^{\prime \prime}\right]$ in the vicinity of the interface

Based on the results of Figs 6-8, Fig. 9 shows the effect of yttria on the macroscopic electrical conductivity of polycrystalline YSZ, for grain sizes larger than $1 \mu \mathrm{m}$. The predicted total electrical conductivity is in excellent agreement with experimental results, as reported by Dixon and co-workers, ${ }^{33}$ Strickler, and Carlson, ${ }^{34}$ and Casselton. ${ }^{35}$ Here, because the oxygen vacancies depletion zone decreases with the addition of yttria, the total electrical conductivity increases, reaching a maximum value in the 7 to 9 mol\% $\mathrm{Y}_{2} \mathrm{O}_{3}$ range in agreement with experiments, ${ }^{34}$ and Kinetic Monte Carlo simulation results. ${ }^{36}$ In the high yttria doping limit, the attractive interactions between $\left[V_{\mathrm{O}}^{\prime \prime}\right]$ and $\left[Y_{\mathrm{Zr}}^{\prime}\right]$ decrease the 
Table 1. Summary of physical parameters used in charged interfacial transitions calculations for YSZ

\begin{tabular}{|c|c|c|c|c|}
\hline Parameter & Symbol & Value & Units & Ref \\
\hline Yttrium defects ionic valence & $Z_{Y}$ & -1 & - & - \\
\hline Mole fraction of electrons & {$[\mathrm{h}]$} & $3.37 \times 10^{-4} \mathrm{P}_{\mathrm{O} 2}^{1 / 4}$ & - & 70,71 \\
\hline Partial pressure of oxygen & $\mathrm{P}_{\mathrm{O} 2}$ & 1 & atm & - \\
\hline Segregation energy of oxygen vacancies for $\Sigma 5$ grain boundary & $f_{V_{0}}^{S}-f_{V_{0}}^{X}$ & -0.5 & $\mathrm{eV}$ & 61,72 \\
\hline Segregation energy of electrons & $f_{\mathrm{e} \prime}^{S^{\prime}}-f_{\mathrm{e} \prime}^{X^{2 r}}$ & -0.1 & $\mathrm{eV}$ & 73 \\
\hline Segregation energy of holes & $f_{\mathrm{h}}^{S}-f_{\mathrm{h}}^{X}$ & -0.1 & $\mathrm{eV}$ & 73 \\
\hline Interaction parameter & $\Omega_{Y_{Z r}^{\prime} V_{0}^{\prime}}$ & -0.13 & $\mathrm{eV}$ & 36 \\
\hline Diffusion coefficient of electrons & $D_{\mathrm{e} \prime}$ & $3.2 \times 10^{-10}$ & $\mathrm{~m}^{2} / \mathrm{s}$ & 74 \\
\hline Diffusion coefficient of holes & $D_{\mathrm{h}}$ & $5.4 \times 10^{-11}$ & $\mathrm{~m}^{2} / \mathrm{s}$ & 74 \\
\hline Order-disorder interfacial thickness & $\delta$ & 1 & $\mathrm{~nm}$ & - \\
\hline Grain boundary energy penalty coefficient & $a=\sqrt{6 \gamma \delta}$ & $6 \times 10^{-5}$ & $(\mathrm{~J} / \mathrm{m})^{1 / 2}$ & 60 \\
\hline Barrier height scaling parameter & $a=\sqrt{\frac{6 \gamma}{\delta}}$ & $6 \times 10^{4}$ & $\left(\mathrm{~J} / \mathrm{m}^{3}\right)^{1 / 2}$ & 60 \\
\hline Structural coupling parameter & $s=\frac{2 a a}{\Delta \theta_{\max }}$ & 9.17 & $\mathrm{~J} / \mathrm{m}^{2}$ & 57 \\
\hline Relative dielectric permittivity of YSZ & $\epsilon_{r}$ & 32 & - & 75 \\
\hline Electrostatic grain boundary potential at critical misorientation for 8YSZ & $\phi_{c}$ & 0.18 & V & - \\
\hline Chemical energy density of disordered region of YSZ & $\Delta f_{\circ}$ & $0.19 \times 10^{9}$ & $\mathrm{~J} / \mathrm{m}^{3}$ & - \\
\hline Electrostatic charge density of disordered region of YSZ & $\rho_{\circ}$ & $0.5 \times 10^{9}$ & $\mathrm{C} / \mathrm{m}^{3}$ & - \\
\hline
\end{tabular}

ionic mobility of oxygen vacancies and lead to a significant decrease in the total conductivity.

Results demonstrate that charged interfaces in polycrystalline cubic YSZ have an important effect on the macroscopic ionic transport properties. Figure 9 shows that in the 5 to $10 \mathrm{~mol} \% \mathrm{Y}_{2} \mathrm{O}_{3}$ doping range, crystallographic disorder in an MS-type interface does not have a strong effect on the electrical conductivity of the grain boundaries in spite of the wide depletion zone of oxygen vacancies in the vicinity of the structural grain boundary. Above $10 \mathrm{~mol} \% \mathrm{Y}_{2} \mathrm{O}_{3}$, the total conductivity associated to MS-type interface is two to three times smaller than that displayed by polycrystals with D-type interface due to the formation of a thick grain boundary core of $\left[V_{0}^{\prime \prime}\right]$, which hinders the ionic transport of $\left[V_{0}^{\prime \prime}\right]$ across the interface because of the insulating effect of $\left[Y_{\mathrm{Zr}}^{\prime}\right]$.

Finally, the developed theory provides a basis to understand the generality of ionic transport properties by correlating the local and microstructural response to the structural and electrochemical phase transitions of the interfaces of ionic polycrystalline ceramics. The application of this formulation enables the development of a mesostructure-level understanding of the microstructureproperties relations of ionic solids, such as those used in SOFCs. While it is beyond the scope of this paper, the current variational formulation sets the stage to understand the fundamentals of other technologically relevant ceramic materials, such as those used in lithium-ion battery applications, dielectric oxides, ferroelectrics, ${ }^{68}$ piezotronics, ${ }^{44}$ and semiconductor applications, ${ }^{43}$ by introducing physically relevant constitutive relationships, $D_{i}=\epsilon_{i j} E_{j}+P_{i}+d_{i j k} C_{j k l m} \epsilon_{l m}$, as proposed in $^{48}$ or as recently proposed by Guyer and co-workers ${ }^{52}$ or Shen et al. ${ }^{45}$

\section{METHODS}

A two-grain, bicrystal YSZ, with a planar grain boundary was modeled by placing the interface at the origin of the laboratory reference system. The normal of the interface was arbitrarily set to point along the $x$-axis. The simulations are conducted at $2000 \mathrm{~K}$ at which the cubic phase of Zirconia is stabilized by adding $\mathrm{Y}_{2} \mathrm{O}_{3}$ in the composition range of $5-25 \% .{ }^{32}$ Without any loss of generality, the segregation energies used in the calculations correspond to a $\Sigma 5$ grain boundary. ${ }^{61}$ YSZ properties are summarized in Table 1. Equation set 4 was solved across a $1 \mu \mathrm{m}$ simulation domain, and discretized into a 10,000 mesh. The partial differential equations were solved by implementing them in FiPy. ${ }^{69}$ The relative tolerance for the convergence was set to $1 \times 10^{-8}$. Each calculation took on the order of one hour of wall time to complete. The macroscopic ionic conductivity of the YSZ polycrystal was calculated by generalizing the model proposed by Guo. ${ }^{30}$ Here, the contributions to the total charge conductivity from each differential element of a representative grain boundary-bicrystal system was integrated to calculate the through-thickness conductivity of YSZ in its polycrystalline form:

$$
\kappa=\frac{L}{\int_{0}^{L} \frac{d x}{\sum_{i=1}^{N} \frac{e^{2} z_{i}^{2}\left[V_{i}^{Z_{i}}\right] D_{i}}{k_{B} T^{T}}}}
$$

The oxygen vacancies diffusivity in YSZ, was set to $D_{V}=9.57 \times 10^{-6}$ $\left[V_{0}^{\prime \prime}\right] \mathrm{e}^{-\frac{2703.57 \times\left[V_{0}\right]+6962.98}{T}} \mathrm{~m}^{2} / \mathrm{s}$ in agreement with Pornprasertsuk and coworkers. ${ }^{36}$

\section{DATA AVAILABILITY}

The simulation data from this study are available upon request.

\section{ACKNOWLEDGEMENTS}

KSNV and REG thank Prof. S. Ramanathan and Prof. J. Greeley for the useful discussions. KSNV and REG are very grateful for the support provided by US ONR N00014- 17-1-2087. 


\section{AUTHOR CONTRIBUTIONS}

KSNV and REG jointly developed the theoretical formulation. KSNV wrote and performed the numerical simulations shown herein. He also compared the calculated results against experimental data available in literature under the guidance of REG.

\section{ADDITIONAL INFORMATION}

Competing interests: The authors declare no competing interests.

Publisher's note: Springer Nature remains neutral with regard to jurisdictional claims in published maps and institutional affiliations.

\section{REFERENCES}

1. Clarke, D. R. On the equilibrium thickness of intergranular glass phases in ceramic materials. J. Am. Ceram. Soc. 70, 15-22 (1980).

2. Clarke, D. R., Shaw, T. M., Philipse, A. P. \& Horn, R. G. Possible electrical double layer contribution to the equilibrium thickness of intergranular glass films in polycrystalline ceramics. J. Am. Ceram. Soc. 76, 1201-1204 (1993).

3. Cahn, J. W. Transition and phase equilibria among grain boundary structures. J. De. Phys. 43, C6-199-C6-213 (1982).

4. Cahn, J. W. Critical point wetting. J. Chem. Phys. 66, 3667-3672 (1977).

5. Kikuchi, R. \& Cahn, J. W. Grain boundaries with impurities in a two-dimensional lattice-gas model. Phys. Rev. B: Condens. Matter Mater. Phys. 36, 418 (1987).

6. Malavasi, L., Fisher, C. A. J. \& Islam, M. S. Oxide-ion and proton conducting electrolyte materials for clean energy applications: Structural and mechanistic features. Chem. Soc. Rev. 39, 4370-4387 (2010).

7. Baram, M., Chatain, D. \& Kaplan, W. D. Nanometer-thick equilibrium films: the interface between thermodynamics and atomistics. Science 332, 206-209 (2011).

8. Tang, M., Carter, W. C. \& Cannon, R. M. Diffuse interface model for structural transitions of grain boundaries. Phys. Rev. B: Condens. Matter Mater. Phys. 73, 024102 (2006).

9. Tang, M., Carter, W. C. \& Cannon, R. M. Grain boundary transitions in binary alloys. Phys. Rev. Lett. 97, 075502 (2006).

10. Dillon, S. J., Tang, M., Carter, W. C. \& Harmer, M. P. Complexion: a new concept for kinetic engineering of materials. Acta Mater. 55, 6208 (2007).

11. Gibbs, J. W. On the equilibrium of heterogeneous substances. Phys. Rev. 44, 108-437 (1878).

12. Luo, J. Grain boundary complexions: the interplay of premelting, prewetting, and multilayer adsorption. Appl. Phys. Lett. 95, 071911 (2009).

13. Rickman, J. M. \& Luo, J. Layering transitions at grain boundaries. Curr. Opin. Solid State Mater. Sci. 20, 225-230 (2016).

14. Luo, J., Wang, H. \& Chiang, Y. M. Origin of solid state activated sintering in $\mathrm{Bi}_{2} \mathrm{O}_{3}$ doped ZnO. J. Am. Ceram. Soc. 82, 916 (1999).

15. Luo, J. Liquid like interface complexion: From activated sintering to grain boundary diagrams. Curr. Opin. Solid State Mater. Sci. 12, 81-88 (2008).

16. Kayyar, A., Qian, H. \& Luo, J. Surface adsorption and disordering in $\mathrm{LiFePO}_{4}$ based battery cathodes. Appl. Phys. Lett. 95, 221905 (2009).

17. Guo, X. \& Waser, R. Space charge concept for acceptor-doped zirconia and ceria and experimental evidences. Solid State lon. 173, 63-67 (2004).

18. Frenkel, J. Kinetic Theory of Liquids. 36 edn, (Oxford University Press, New York, 1946).

19. Lehovec, K. Space-charge layer and distribution of lattice defects at the surface of ionic crystals. J. Chem. Phys. 21, 1123 (1953).

20. Eshelby, J. D., Newey, C. W. A., Pratt, P. L. \& Lidiard, A. B. Charged dislocations and the strength of ionic crystals. Philos. Mag. 3, 75-89 (1958).

21. Kliewer, K. L. \& Koehler, J. S. Space charge in ionic crystals. I. General approach with application to $\mathrm{NaCl}$. Phys. Rev. 140, A1226-A1240 (1965).

22. Maier, J. lonic-conduction in space charge regions. Prog. Solid. State 23, 171-263 (1995).

23. Guo, X., Sigle, W. \& Maier, J. Blocking grain boundaries in yttria-doped and undoped ceria ceramics of high purity. J. Am. Ceram. Soc. 86, 77-87 (2003).

24. Kim, S. \& Maier, J. On the conductivity mechanism of nanocrystalline ceria. J. Elec. Soc. 149, J73-J83 (2002).

25. Luo, J. Interfacial engineering of solid electrolytes. J. Mater. 1, 22-32 (2015).

26. Mott, N. F. Theory of crystal rectifiers. Proc. Roy. Soc. (Lond.) A. 171, 27 (1939).

27. Schottky, W. Z. Zur halbleitertheorie der sperrschicht- und spitzengleichrichter. Physik 113, 367 (1939).

28. Guo, X. \& Waser, R. Electrical properties of the grain boundaries of oxygen ion conductors: acceptor-doped zirconia and ceria. Progress. Mater. Sci. 51, 151-210 (2006).

29. Ma, C. et al. Atomic scale origin of the large grain boundary resistance in perovskite Li-ion-conducting solid electrolytes. Energy Environ. Sci. 7, 1638 (2014).
30. Guo, X. Physical origin of the intrinsic grain boundary resistivity of stabilized zirconia: role of the space-charge layers. Solid State Ion. 81, 235-242 (1995).

31. Rheinheimer, W. \& Hoffmann, M. J. Grain growth in perovskites: what is the impact of boundary transitions? Curr. Opin. Solid State Mater. Sci. 20, 286-298 (2016).

32. Scott, H. G. Phase relationships in the zirconia-yttria system. J. Mater. Sci. 10, 1527-1535 (1975).

33. Dixon, J. M., LaGrange, L. D., Merten, U., Miller, C. F. \& Porter, J. T. li Electrical resistivity of stabilized zirconia at elevated temperatures. J. Electrochem. Soc. 110, 276-280 (1963).

34. Strickler, D. W. \& Carlson, W. G. Ionic conductivity of cubic solid solutions in the system CaO- $\mathrm{Y}_{2} \mathrm{O}_{3}-\mathrm{ZrO}_{2}$. J. Am. Ceram. Soc. 47, 123-127 (1964).

35. Casselton, R. E. W. Low field dc conduction in yttria-stabilized zirconia. Phys. Status Solidi A 2, 571-585 (1970).

36. Pornprasertsuk, R., Ramanarayanan, P., Musgrave, C. B. \& Prinz, F. B. Predicting ionic conductivity of solid oxide fuel cell electrolyte from first principles. J. Appl. Phys. 98, 103513 (2005)

37. Aoki, M., Chiang, Y. M., Kosacki, I., Lee, L. J. R. \& Liu, H. T. Y. Solute segregation and grain-boundary impedance in high-purity stabilized zirconia. J. Am. Ceram. Soc. 79, 1169-1180 (1996).

38. Yoshida, H., Yokoyama, K., Shibata, N., Ikuhara, Y. \& Sakura, T. High-temperature grain boundary sliding behavior and grain boundary energy in cubic zirconia bicrystals. Acta Mater. 52, 2349-2357 (2004).

39. Shibata, N., Oba, F., Yamamoto, T. \& Ikuhara, Y. Structure, energy and solute segregation behavior of [110] symmetric tilt grain boundaries in yttria-stabilized cubic zirconia. Philos. Mag. Lett. 84, 2381-2415 (2004).

40. Lee, H. B., Prinz, F. B. \& Cai, W. Atomistic simulations of surface segregation of defects in solid oxide electrolytes. Acta Mater. 58, 2197-2206 (2010).

41. Lee, H. B., Prinz, F. B. \& Cai, W. Atomistic simulations of grain boundary segregation in nanocrystalline yttria-stabilized zirconia and gadolinia-doped ceria solid oxide electrolytes. Acta Mater. 61, 3872-3887 (2013).

42. Rodewald, S., Fleig, J. \& Maier, J. Resistance degradation of iron doped strontium titanate investigated by spatially resolved conductivity measurements. J. Am. Ceram. Soc. 83, 1969-1976 (2000)

43. Cao, Y., Bhattacharya, S., Shen, J., Randall, C. A. \& Chen, L. Q. Role of polaron hopping in leakage current behavior of a $\mathrm{SrTiO}_{3}$ single crystal. J. Appl. Phys. 114 224102 (2013).

44. Sluka, T., Tagantsev, A. K., Damjanovic, D., Gureev, M. \& Setter, N. Enhanced electromechanical response of ferroelectrics due to charged domain walls. Nat Comm. 3, 748 (2012)

45. Shen, Z. H. et al. Space charge effects on the dielectric response of polymer nanocomposites. Appl. Phys. Lett. 111, 092901 (2017).

46. Jeong, D. S., Schroeder, H. \& Waser, R. Mechanism for bipolar switching in a Pt/ $\mathrm{TiO}_{2} / \mathrm{Pt}$ resistive switching cell. Phys. Rev. B 79, 195317 (2009).

47. Hart, E. W. Thermodynamics of inhomogeneous systems. Phys. Rev. 113, 412-416 (1959).

48. Garca, R. E., Bishop, C. M. \& Carter, W. C. Thermodynamically consistent variational principles with applications to electrically and magnetically active systems. Acta Mater. 52, 11-21 (2004).

49. Vikrant, K. S. N., Chueh, W. C. \& Garca, R. E. Charged interfaces: electrochemical and mechanical effects. Energy Environ. Sci. 11, 1993-2000 (2018).

50. DeHoff, R. T. Thermodynamics in Materials Science. 2 edn, (McGraw-Hill, New York, 1993).

51. Bishop, C. M., Garca, R. E. \& Carter, W. C. Effect of charge separation on the stability of large wavelength fluctuations during spinodal decomposition. Acta Mater. 51, 1517-1524 (2003).

52. Guyer, J. E., Boettinger, W. J., Warren, J. A. \& McFadden, G. B. Phase field modeling of electrochemistry I: Equilibrium. Phys. Rev. E. 69, 021603 (2004).

53. Kobayashi, R., Warren, J. A. \& Carter, W. C. A continuum model of grain boundaries. Phys. D. 140, 141-150 (2000).

54. Pusztai, T., Bortel, G. \& Gránásy, L. Phase field theory of polycrystalline solidification in three dimensions. Europhys. Lett. 71, 131 (2005).

55. Kobayashi, R. \& Warren, J. A. Modeling the formation and dynamics of polycrystals in 3D. Phys. A 356, 127-132 (2005).

56. Gránásy, L. et al. Phase-field modeling of polycrystalline solidification: from needle crystals to spherulites-a review. Metall. Mater. Trans. A 45, 1694-1719 (2014).

57. Warren, J. A., Kobayashi, R., Lobkovsky, A. E. \& Carter, W. C. Extending phase field models of solidification to polycrystalline materials. Acta Mater. 51, 6035-6058 (2003).

58. Cahn, J. W. \& Hilliard, J. E. Free energy of a nonuniform system. I. Interfacial free energy. J. Chem. Phys. 28, 258-267 (1958).

9. Cahn, J. W. On spinodal decomposition. Acta Metall. 9, 795-801 (1961).

60. Boettinger, W. J., Warren, J. A., Beckermann, C. \& Karma, A. Phase-field simulation of solidification. Annu. Rev. Mater. Res, 32, 163-194 (2002). 
61. Romero, R. L. G., Melendez, J. J., Garca, D. G., Cumbrera, F. L. \& Rodriguez, A. D. Segregation to the grain boundaries in YSZ bicrystals: a molecular dynamics study. Solid Sate Ion. 237, 8-15 (2013).

62. Zhu, J. Z., Liu, Z. K., Vaithyanathan, V. \& Chen, L. Q. Linking phase-field model to CALPHAD: An application to precipitate shape evolution in Ni-base alloys. Scr. Mater. 46, 401-406 (2002).

63. Ozturk, K. et al. Linking first-principles energetics to CALPHAD: An application to thermodynamic modeling of the Al-Ca binary system. Metall. Mater. Trans. A 36A, 5-13 (2005).

64. Jackson, J. D. Classical Electrodynamics (Wiley, New Jersey, 1998)

65. Gelfand, I. M. \& Fomin, S. V. Calculus of Variations (Dover Publications, USA, 1991).

66. Gouy, M. G. Sur la constitution de la charge electrique a la surface d'un electrolyte. J. Phys. Radium 3, 457-468 (1910).

67. Chapman, D. L. A contribution to the theory of electrocapillarity. Philos. Mag. 6, (1913).

68. Torres-Matheus, O. A., Garca, R. E. \& Bishop, C. M. Phase coexistence near the polymorphic phase boundary. Acta Mater. 164, 577-585 (2019).

69. Guyer, J. E., Wheeler, D. \& Warren, J. A. FiPy: partial differential equations with python. Comput. Sci. Eng. 11, 615 (2009).

70. Kiukkola, K. \& Wagner, C. Measurements on galvanic cells involving solid electrolytes. J. Electrochem. Soc. 104, 379-386 (1957).

71. Casselton, R. E. W. \& Scott, J. C. Conduction mechanism in yttria stabilized zirconia. Phy. Lett. 25A, 264-265 (1967).

72. Xia, X., Catlow, C. R. A. \& Oldman, R. Computational modeling study of bulk and surface of yttria-stabilized cubic zirconia. Chem. Mater. 21, 3576-3585 (2009)
73. Heiroth, S., Ghisleni, R., Lippert, T., Michler, J. \& Wokaun, A. Optical and mechanical properties of amorphous and crystalline yttria-stabilized zirconia thin films prepared by pulsed laser deposition. Acta Mater. 59, 2330-2340 (2011).

74. Huang, X. J. \& Weppner, W. Electrochemical transient investigations on the diffusion of minority charge carriers in YSZ doped by transition metal oxides. lonics 5, 91-99 (1999).

75. Thompson, D. P. \& Dickins, A. M. The dielectric properties of zirconia. J. Mater. Sci. 27, 2267-2271 (1992).

(i) Open Access This article is licensed under a Creative Commons Attribution 4.0 International License, which permits use, sharing, adaptation, distribution and reproduction in any medium or format, as long as you give appropriate credit to the original author(s) and the source, provide a link to the Creative Commons license, and indicate if changes were made. The images or other third party material in this article are included in the article's Creative Commons license, unless indicated otherwise in a credit line to the material. If material is not included in the article's Creative Commons license and your intended use is not permitted by statutory regulation or exceeds the permitted use, you will need to obtain permission directly from the copyright holder. To view a copy of this license, visit http://creativecommons. org/licenses/by/4.0/.

(c) The Author(s) 2019 